\title{
Kinder, sammelt Knochen!
}

\section{Lehr- und Propagandamittel zur Behandlung des Themas Knochenverwertung an deutschen Schulen im „Dritten Reich"}

\author{
Elisabeth Vaupel und Florian Preiß
}

Children, Collect Bones! Teaching Aids and Propaganda Material on Bone-Collections and Bone-Utilisation Used in German Schools During the "Third Reich"

In the nineteenth and early twentieth centuries bones were an essential raw material for the German chemical industry, vital to the production of fertilizer, glue, gelatine, soap and other products. As most of this material was imported, the German school system during the "Third Reich" took the utilisation of bones as an example to illustrate the relevance of the four-year plan of 1936 and its policy of economic self-sufficiency. The school children were encouraged to collect bones from domestic sources and bring them to the collecting points in the schools. Several NS-institutions developed a variety of teaching aids and materials to support school education on this economically and politically important topic. Focussing on the example of bone-utilisation, this paper examines the messages and intentions of these educational materials. It also demonstrates how even apparently ideologically unbiased school subjects, such as chemistry, were instrumentalised for the political indoctrination of the pupils.

Keywords: "Third Reich", School education, Teaching aids, Classroom wall chart, Waste collections, Bones

Knochen waren im 19. und frühen 20. Jahrhundert ein unverzichtbarer, größtenteils importabhängiger Rohstoff der chemischen Industrie, die daraus Düngemittel, Tierfutter, Leim, Gelatine, Seife und andere Produkte herstellte. Das Thema Knochenverwertung wurde im Schulunterricht der NS-Zeit genutzt, um Jugendlichen die Relevanz des Vierjahresplans und der deutschen Autarkiepolitik zu verdeutlichen und sie zu motivieren, sich im Rahmen der Altstoffsammlungen an der heimischen Erfassung dieses Rohmaterials zu beteiligen. Diverse NS-Instanzen hatten ein differenziertes Spektrum von Lehrmitteln erarbeitet, um die Behandlung dieses Themas in der Schule zu unterstützen. Der Artikel untersucht, welche Botschaften diese damals hochmodernen Unterrichtsmedien vermittelten und zeigt, wie auch weniger ideologieanfällig scheinende Fächer, etwa der Chemieunterricht, politisch instrumentalisiert wurden.

Schlüsselwörter: „Drittes Reich“, Schulunterricht, Lehrmittel, Schulwandbild, Altmaterialsammlung, Knochen

Der Schulunterricht in der NS-Zeit und die Ideologisierung von Bildung und Erziehung werden seit Jahrzehnten erforscht. ${ }^{1}$ Mittlerweile liegen nicht nur Arbeiten über einzelne Schultypen und -formen, sondern auch über einzelne Unterrichtsfächer vor, wobei sich das Interesse zunächst auf die

Electronic Supplementary Material (ESM)

Das gesamte Bildmaterial, das im Artikel erwähnt wird, ist als Electronic Supplementary Material (ESM) in der Online-Version dieses Artikels enthalten: https://doi.org/10.1007/s00048-018-0194-y. 
stark ideologiegeprägten Fächer Deutsch, Geschichte, Erdkunde und im naturwissenschaftlichen Bereich die Biologie konzentrierte (Weiss 1994; Bäumer-Schleinkofer 1995). Es gibt aber auch erste Untersuchungen über den weniger ideologieanfällig scheinenden Physik- und Chemieunterricht (Brämer \& Kremer 1980; Stoya 2002). Dieser Aufsatz beschäftigt sich mit einem Detailaspekt des Schulunterrichts in der Zeit des „Dritten Reichs“: den zahlreich überlieferten Lehrmitteln zum Thema Knochenverwertung. Verschiedene Schulbücher, vor allem aber die damals beliebten Lehrtafeln, ${ }^{2}$ Schülerarbeitsblätter, Diaserien und Unterrichtsfilme belegen, wie intensiv und multimedial dieses Thema seit 1937 behandelt wurde. Da diese mittlerweile ungebräuchlichen Anschauungsmittel heute in Museen und Spezialsammlungen aufbewahrt werden, wurden sie von der historischen Forschung bisher nicht beachtet. Die Requirierung und Verwertung von Altstoffen, die in der NS-Zeit nicht nur vom Deutschen Reich und dessen Verbündeten, sondern auch von den Alliierten und den neutralen Staaten praktiziert wurde, ${ }^{3}$ erhält durch den hier verfolgten Ansatz, die Vielfalt dieser Unterrichtsmittel vor dem Hintergrund der wirtschaftlichen Bedeutung des Rohstoffs Knochen zu analysieren, neue Facetten und schärfere Konturen. Im Folgenden wird zunächst die Relevanz der den Schülern aufgebürdeten Knochensammlungen für die deutsche Autarkieund Rüstungspolitik dargestellt und gezeigt, wie diese Aktivitäten seitens des Schulunterrichts mit Hilfe diverser Lehr- und Propagandamaterialien begleitet und unterstützt wurden; dann wird die (kriegs)wirtschaftliche Bedeutung verschiedener Produkte diskutiert, die im „Dritten Reich“ aus dem historisch bislang noch kaum gewürdigten Industrierohstoff Knochen hergestellt wurden.

Um ein einheitliches nationalsozialistisches Erziehungssystem aufzubauen, war 1934 das Reichsministerium für Wissenschaft, Erziehung und Volksbildung geschaffen worden, kurz Reichserziehungsministerium genannt (Nagel 2012). Durch seine Gründung war zum ersten Mal in der deutschen Schulgeschichte eine zentralstaatliche Instanz für die Bildungspolitik zuständig geworden. Die schulpolitischen Aktivitäten des neuen Ministeriums konsolidierten sich allerdings erst ab 1937, als sukzessiv reichseinheitliche neue Lehrpläne für die einzelnen Schultypen erschienen (Stoya 2002: 26 f.). Wenige Monate zuvor, im Herbst 1936, war der für die NSWirtschaftspolitik wichtige Vierjahresplan bekannt gegeben worden, der die Lehrinhalte an den Schulen nachhaltig prägen sollte (Anonym 1937; Hartmann 1938; Wolter 1938). Innerhalb von vier Jahren sollte sich die deutsche Wirtschaft von Auslandsimporten weitgehend unabhängig und damit kriegsfähig machen. Da sich im Ersten Weltkrieg gezeigt hatte, wie verwundbar das ressourcenarme, auf Rohstoffimporte angewiesene Deutsche Reich im Kriegsfall war, verfolgte der NS-Staat eine dezidierte Autar- 
kiepolitik. Wirtschaft und Industrie wurden aufgefordert, die Verwendung einfuhrabhängiger Roh- und Werkstoffe auf das Nötigste zu beschränken und möglichst auf Ersatz aus inländischen Ressourcen auszuweichen. Um den Jugendlichen $\mathrm{zu}$ verdeutlichen, dass die Umsetzung des Vierjahresplans auch ihnen neue Pflichten abverlangte, wurde die Rohstoffversorgung Deutschlands in der Schule unabhängig von Schultyp und Jahrgangsstufe seit 1937 in vielen Fächern behandelt, besonders im Chemieunterricht (Stoya 2002: 13f.).

\section{Der Vierjahresplan im Unterricht}

Das 1938 vom Reichserziehungsministerium veröffentlichte Werk Erziehung und Unterricht in der Höheren Schule attestierte dem Chemieunterricht großen „erzieherischen Wert“, vor allem aufgrund der Schulversuche und der durch die Schule organisierten Besichtigungen chemischer Betriebe. Das Ministerium forderte, dass der Chemieunterricht Interesse an volkswirtschaftlichen Fragen wecken und zugleich vermitteln solle, dass Naturwissenschaft und Technik den „wirtschaftlichen und kulturellen Hochstand“ des deutschen Volkes sicherten; er solle zeigen, wie die wissenschaftliche und technische Chemie der deutschen Industrie „neue wertvolle Roh- und Werkstoffe aus heimatlichen Stoffen“ zur Verfügung stellten, die Jugend zu Sparsamkeit im Umgang mit den in Deutschland vorhandenen Stoffen erziehen und „zum Verständnis der Gegenwartsfragen" beitragen. Auch müsse er verdeutlichen, welche zentrale Rolle die Chemie spiele, um die Ernährung und die Verteidigungsfähigkeit des Reichs zu sichern (Erziehung und Unterricht in der Höheren Schule 1938: 165-172). Die damaligen Chemiebücher zeigen, dass diese Vorstellungen durch eine verstärkte Ausrichtung der Lerninhalte auf nationale und volkswirtschaftliche Aspekte umgesetzt wurden. Die Behandlung der deutschen Chemiegeschichte (Bäumer-Schleinkofer 1994), der Wehrchemie und der Rohstoffsituation im Reich belegen diese Politik (Beier 1934; Gölz \& Jansen 1990; Gölz \& Jansen 1993). Die in den Schulbüchern enthaltenen Daten über die deutsche Inlands- sowie die Auslandsproduktion bestimmter Rohstoffe lenkten die Aufmerksamkeit der Schüler und Schülerinnen immer wieder auf den Vierjahresplan. Da Koedukation im NSStaat abgelehnt wurde und die Mädchenschulen ihre Schülerinnen durch verstärktes Thematisieren ernährungs- und hauswirtschaftlicher Aspekte der Chemie auf ihre künftigen Aufgaben als Hausfrau und Mutter vorbereiten sollten, fällt die Behandlung der Wehrchemie und bestimmter Facetten der Vierjahresplanchemie vor allem in den Schulbüchern für Jungenschu- 
len auf, besonders denen für den mathematisch-naturwissenschaftlichen Zweig (Dörmer \& Dörmer 1939). Dieser Schultyp gestand dem naturwissenschaftlichen Unterricht (Physik und Chemie) mehr Wochenstunden zu (drei Stunden von Klasse 10 bis 13) als die Mädchenschulen (drei Stunden in Klasse 10 und 12) oder die klassischen Gymnasien (zwei Stunden in Klasse 10) und erlaubte daher eine intensivere Behandlung des Lehrstoffs (Erziehung und Unterricht in der Höheren Schule 1938: 25-31; Stoya 2002: 5).

Dem Experimentalunterricht kam in der NS-Zeit besondere Bedeutung zu, weil er Geschicklichkeit, Beobachtungsgabe, Gewissenhaftigkeit, Reinlichkeit, Ordnungssinn sowie die Fähigkeit zur präzisen Erledigung einer Aufgabe schulte und damit Kompetenzen trainierte, die auch der Wehrerziehung dienten. Vierjahresplanrelevante Themen wurden daher gern im Schulversuch behandelt (Erziehung und Unterricht in der Höheren Schule 1938: 166f.; Stoya 2002: 154-168). Üblicherweise fanden die Schülerexperimente im Rahmen der vom Reichserziehungsministerium eingeführten naturwissenschaftlich-mathematischen Arbeitsgemeinschaften statt, die engagierte Lehrer zusätzlich zum Pflichtunterricht anboten. Die Versuche sollten die Jugendlichen einerseits in die wissenschaftliche Forschungspraxis einführen, zugleich aber „lebensnah“, „praktisch verwertbar" und „für die Gemeinschaft nützlich" sein (Erziehung und Unterricht in der Höheren Schule 1938: 3; 204-206). Um den Lehrern die Gestaltung des Experimentalunterrichts zu erleichtern, erschienen in den späten 1930er Jahren viele Bücher mit Versuchen zur Wehr- und Vierjahresplanchemie. ${ }^{4}$ Eines davon sei hier hervorgehoben, weil es unter anderem Experimente zum Thema Knochenverwertung enthielt: die Schulversuche mit deutschen Roh-und Werkstoffen, die der an der Hochschule für Lehrerfortbildung in Leipzig tätige Walter Haferkorn zusammengestellt hatte (Haferkorn 1938). Ergänzend dazu hatte er mit seinem Kollegen Herbert Priemer eine aus 31 Tafeln bestehende Serie mehrfarbiger Schulwandbilder konzipiert, die im gleichen Verlag wie das Buch publiziert wurde (Haferkorn \& Priemer 1937-1939). Mit diesen aufeinander abgestimmten Unterrichtsmaterialien konnten alle vierjahresplanrelevanten Roh- und Werkstoffe im Unterricht behandelt werden. Die Tafeln veranschaulichten vor allem die Verfahren bei der Herstellung und Verarbeitung eines bestimmten Rohstoffs. Das erste Bild der Serie erschien im Mai 1937 und behandelte den Rohstoff Knochen und seine Verwertung (siehe Abb. 1). Geeignete Knochenversuche (Kahn 1923/24; Pröbsting 1936; Hessenland 1938: 71-73) standen auch in Haferkorns Experimentierbuch an erster Stelle. ${ }^{5}$ Eine Broschüre mit einschlägigen Versuchen für die Volksschule (Garz 1939) sowie die Publikation weiterer Knochenexperimente in den renommierten Unterrichtsblättern für Mathematik und Naturwissenschaften (Zeitler 1940) belegen, wie 


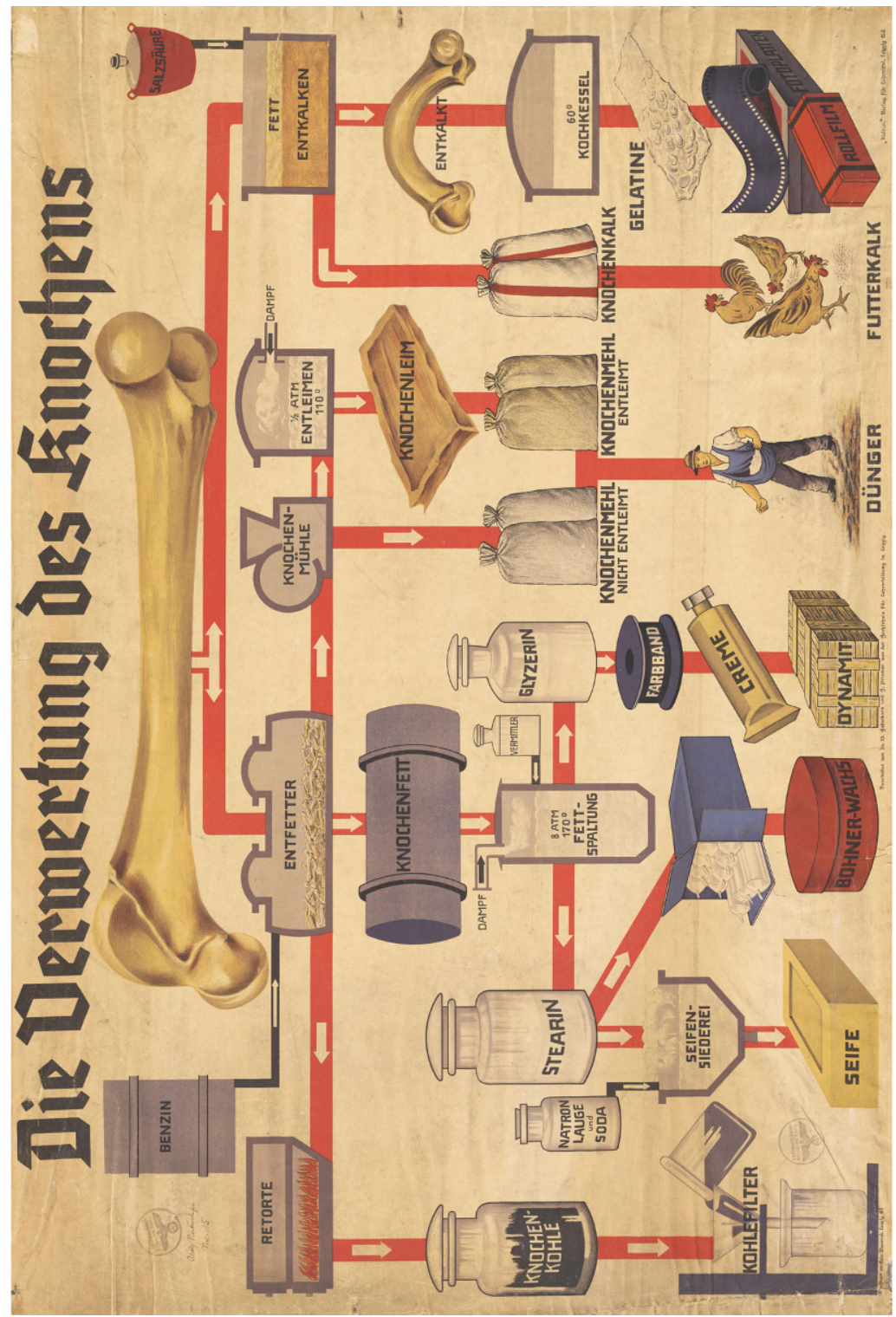

Abb. 1 Lehrtafel zum Thema „Knochenverwertung” aus der von Haferkorn und Priemer herausgegebenen Serie "Anschauungstafeln zur deutschen Nationalwirtschaft" für Berufs-, Berufsfach- und Fachschulen sowie den Chemie- und Technikunterricht an Oberrealschulen, 1937, Farblithografie auf Papier, $67 \times 97,5 \mathrm{~cm}$, Forschungsstelle Historische Bildmedien, Universität Würzburg. Die Serie enthielt noch zwei weitere Tafeln zum Thema Altmaterialverwertung (Altmetall; Lumpen und Altpapier). 
sehr sich die Pädagogen um die experimentelle Behandlung gerade dieses Themas bemühten.

\section{Knochensammlungen}

Zur Einordnung dieser Befunde muss auf die prekäre Rohstofflage im Reich und die Bedeutung des Rohmaterials Knochen eingegangen werden. Das Deutsche Reich hatte bereits im Ersten Weltkrieg drei Strategien verfolgt, um den der britischen Seeblockade geschuldeten Rohstoffmangel zu kompensieren: Sparsamkeit im Umgang mit knappen Materialien, Ausweichen auf Ersatzstoffe, die sich aus heimischen Ressourcen synthetisieren ließen und eine möglichst vollständige Nutzung aller im Inland erfassbaren Alt- und Abfallmaterialien. Diesen Strategien kam in der NS-Zeit abermals große Bedeutung zu, hinsichtlich der Knochen vor allem den letztgenannten. Schon 1934 hatte das NS-Regime begonnen, die vorhandenen Strukturen der Altmaterial- und Abfallverwertung auszubauen: Gummi-, Asbest-, Leder-, Textil- und Holzabfälle, Altöl, Altpapier, Altmetall, Glas und andere Wertstoffe wurden möglichst sortenrein gesammelt und einer Verwertung zugeführt. Wie schon im Ersten Weltkrieg galt auch im „Dritten Reich“ der Hausmüll einschließlich der Küchenabfälle als wertvolle heimische Ressource, aus der alles Verwertbare, einschließlich der Knochen, aussortiert werden sollte. Um die Kriegswichtigkeit dieses Rohstoffs, der schon 1917/18 von Hausfrauen und Schülern gesammelt worden war, zu erklären, hatte das von der Obersten Heeresleitung gegründete Bildund Filmamt (Bufa) 1918 einen Kurzfilm mit dem Titel Sammelt Knochen produziert. ${ }^{6}$ Diese Maßnahmen griff Hermann Göring in seiner Funktion als Beauftragter für den Vierjahresplan zwanzig Jahre später wieder auf. Um zu verhindern, dass das Deutsche Reich noch einmal so unvorbereitet in einen Krieg zog wie 1914, stellte sich der nationalsozialistische Staat von langer Hand auf den Kriegsfall ein und schuf deshalb schon 1937 eine Institution, die die Verwertung von Abfallstoffen organisieren sollte: den Reichskommissar für Altmaterialverwertung. Statistische Erhebungen hatten gezeigt, dass nur etwa ein Fünftel der im Reich anfallenden Knochen industriell genutzt wurden; über 70 Prozent gingen verloren, weil sie nicht gesammelt wurden (Wimmer 1937: 5 f; Gerhardt \& Höfner 1942: 34-36). Da die vom Altstoffhandel erfassten Knochen den Industriebedarf jedoch nicht deckten, musste jedes Jahr zusätzliches Material aus dem Ausland eingeführt werden. Um die Devisenausgaben für diese Importe einzusparen oder wenigstens zu reduzieren, sollten ab 1937 zusätzlich zu den Knochenabfällen aus Abdeckereien, Schlachthöfen, Metzgereien und 
der Fleischwarenindustrie auch jene aus Privathaushalten, Betriebskantinen und Gaststätten gesammelt werden, die bisher ungenutzt im Müll verschwunden waren. Modellrechnungen zufolge sollte sich die Requirierung dieser Kleinstmengen rentieren (Ziegelmayer 1936: 136; Ziegelmayer: 1941: 196). Auf die Gesamtzahl aller Haushalte hochgerechnet stellten sie laut NS-Propaganda „Millionenwerte“ dar (Köstering 2003). Anders als im Ersten Weltkrieg wurde die Knochenerfassung nun aber nicht mehr in erster Linie den Hausfrauen überlassen, sondern vor allem den Schülerinnen und Schülern aufgebürdet, wobei darauf spekuliert wurde, über die Kinder auch die Mütter zu motivieren (Anonym 1942j). Auf Grundlage dieser Überlegungen wurden die Schülerinnen und Schüler durch einen im Januar 1937 veröffentlichten Erlass des Reichserziehungsministeriums dazu aufgefordert, die Abfallknochen, die in den Haushalten anfielen, in der Schule abzugeben. Nach den Zäsuren von 1936 (Verkündung des Vierjahresplans) und 1937 (Beginn der schulischen Knochensammlungen) stellte der Kriegsausbruch 1939 die dritte Zäsur in der Geschichte der Rohstofferfassung durch die Schülerinnen und Schüler dar. Sie mussten fortan nicht nur die Arbeitskräfte ersetzen, die dem Rohproduktengewerbe durch die „Arisierung“ der oft in „jüdischem“ Besitz befindlichen Altmaterialhandlungen verlorengegangen waren, sondern auch die Lücken füllen, die nach Kriegsbeginn durch die Einberufungen entstanden. Seit Herbst 1939 lasteten die Knochensammlungen fast ausschließlich auf den Schultern von jugendlichen Personen, die das Sammeln kostenlos erledigten. Dass sich die Hitlerjugend (HJ) und die Sturmabteilung (SA) immer wieder an Stoßaktionen beteiligten, änderte nichts an der grundsätzlichen Umverteilung der Lasten (Miller 2014: 36-38).

\section{Prämiensystem}

Seit seiner Etablierung machte der Reichskommissar für Altmaterialverwertung mit Vorträgen, Presseartikeln, Aufrufen, Filmen, Rundfunksendungen und Broschüren auf die Relevanz der Altmaterialsammlungen aufmerksam (Hertenstein 1939: 280). Nach Kriegsbeginn wurden die Werbeaktivitäten intensiviert. Ein kostenlos verteilter Wandkalender erinnerte die Mütter und schulpflichtigen Kinder daran, die Knochen an den Sammeltagen bei dem zum „Altstoffwart“ bestimmten Schüler oder beim „Altstofflehrer" der Schule abzuliefern. ${ }^{7}$ Dort wurde das Material gewogen und das Resultat dokumentiert. Dann kamen die Knochen in einen Sammelbehälter, der regelmäßig von einem Altmaterialhändler geleert wurde. Dass die Schule somit als Vorsammelstelle für den professionellen Altmaterial- 
handel missbraucht wurde, stieß bei vielen Lehrern auf Vorbehalte (Krause 1943). Regimetreue Pädagogen rechtfertigten dies aber wie folgt:

Sie [die Knochensammlung] bereitet Unannehmlichkeiten für Lehrer und Schüler [...]. Sie kostet vielleicht auch Zeit, die dem Unterricht verloren geht. Aber es wäre doch grundverkehrt, darüber zu klagen. Handelt es sich doch nicht um etwas Schulfremdes, sondern um eine Maßnahme, die wesentlich mit zur gegenwärtigen Schulerziehung gehört. Oder glaubt man vielleicht, mehr getan zu haben, wenn durch Rechenaufgaben und andere Belehrungen allein die Notwendigkeit der Sammlung von Knochen „durchgenommen“ worden ist, ohne dass sie wirklich ausgeübt wurde? Hier scheiden sich die Geister: Der eine denkt noch völlig in Vorstellungen rein formaler Bildung; der andere weiß, dass Bildung nur fruchtbar werden kann, wenn sie eingegliedert ist in politische Erziehung (Schwierskott 1938: 551-552).

Die Knochensammlungen wurden nach Kriegsbeginn wirtschaftlich immer wichtiger, ${ }^{8}$ weil die Materiallieferungen aus Übersee ausfielen. Seit Februar 1940 mussten die Schülerinnen und Schüler außer Knochen auch alle anderen in den Haushaltungen anfallenden Alt- und Abfallstoffe sammeln. 1941 setzte der Reichskommissar für Altmaterialverwertung mit der Schaffung eines Punkte- und Prämiensystems, das die vierte und letzte Zäsur in der Geschichte der schulischen Wertstofferfassung markierte, materielle Anreize, um den Sammeleifer der Kinder anzuspornen (Thomsen 1943: 39; Schildt 2005: 302). Seit jenem Jahr schrieb der „Altstofflehrer“ einzelnen Schülerinnen und Schülern oder Klassen bei Ablieferung einer bestimmten Menge eines bestimmten Altstoffs Punkte gut. Bei dieser Vorgehensweise zeigte sich ganz von selbst, ob und inwiefern die allseits erwarteten Mindestabgabemengen erfüllt worden waren. Da sich ein Kilo Knochen relativ leicht sammeln ließ, während es erheblich schwerer fiel, die gleiche Gewichtsmenge Lumpen zusammenzubekommen, wurde die Abgabe eines Kilos Alttextilien mit fünf Punkten am höchsten bewertet; dann folgten die mit drei Punkten relativ hoch bewerteten Knochen, die ebenso viel zählten wie Buntmetall; mit zwei Punkten stand das Altpapier an dritter Stelle; nur einen Punkt gab es dagegen für ein Kilo Eisenschrott (Steinberg 2008: 50 f.; Brockmann 1994: 121f.; Sagemüller 1994: 232). Bei Erreichen einer bestimmten Punktzahl winkte eine Prämie, beispielsweise ein handschriftlich signiertes Göring-Bild oder eine Urkunde. Es wurden aber auch Sachpreise wie Taschenkalender, Bleistifte, ${ }^{9}$ Bücher, Globen, Zirkelkästen ${ }^{10}$ oder Bücher mit politischem Inhalt verteilt (Wüstenfeld 2002: 29; Barwich 2003: 38 f.). Die reichsbesten Schulen erhielten eine Plastik des Bildhauers Walter Lerche, die Jungenschulen einen Fußballspieler, die Mädchenschulen einen Mädchenkopf (Anonym 1942). Die prestigeträch- 
tigste Auszeichnung einzelner Schülerinnen und Schüler waren Rundfunkapparate (Anonym 1943d) oder eine dreitägige Reise nach Berlin, bei der, falls möglich, sogar ein Treffen mit Göring arrangiert wurde. Erfolgreiche „Altstofflehrer“ erhielten als Anerkennung eine „künstlerisch wertvolle“ Urkunde mit eigenhändiger Unterschrift des Reichskommissars (Anonym 1943d). In der Tat konnten die erfassten Knochenmengen von 1937 bis 1944 kontinuierlich gesteigert werden, trotz der bei Kriegsausbruch eingeführten Fleischrationierung (Nadolni 1944) und trotz des Umstandes, dass sich sogar 1943 noch nicht alle größeren Betriebe (Krankenhäuser, Gast- und Verpflegungsstätten) an den Sammlungen beteiligten (Anonym 1943a). Seit 1944 gingen die Ergebnisse wegen der schlechter werdenden Versorgungslage allerdings zurück, was zu einer Anpassung des Prämiensystems führte: für fünf Kilo Sammelknochen, die auch pfundweise abgeliefert werden konnten, erhielt man nun einen Bezugsschein, der zum Kauf eines Stücks Kernseife berechtigte - eine angesichts der Seifenrationierung attraktive Belohnung. ${ }^{11}$ Das Reichserziehungsministerium nahm die schulischen Knochensammlungen sogar wenige Monate vor Kriegsende noch so ernst, dass es Schulen, die kriegsbedingt schlossen, Ende Januar 1945 von der Altstofferfassung befreite; jene, die den Unterrichtsbetrieb aufrechterhalten konnten, wurden verpflichtet, diese Ausfälle durch umso eifrigeres Sammeln zu kompensieren (Reichsminister für Wissenschaft, Erziehung und Volksbildung 1945: 14).

\section{Lehrmittel für die Volksschule}

Um den Kindern den Sinn und Zweck der Knochensammlungen zu erklären, wurde das Thema Knochenverwertung mehrfach in der Schule behandelt, beginnend in der Volksschule. Zum Knochensammeln wurden nämlich schon die ABC-Schützen herangezogen, trotz der von manchen Eltern vorgetragenen Bedenken wegen möglicher Gesundheitsbeeinträchtigungen: in der Tat waren die Sammelstellen unhygienische Orte, an denen es stank (Teweleit 1993: 43 f; Vorländer 2000: 49) und sogar zu Rattenplagen kam. ${ }^{12}$ Der Reichsausschuss für volkswirtschaftliche Aufklärung (RVA), eine dem Reichspropagandaministerium unterstellte Institution, die unter anderem die Verwertung "deutscher Roh- und Werkstoffe" propagieren sollte, hatte 1939 eine kindgerecht illustrierte Lehrtafel mit der Aufforderung „Wir sammeln Knochen! Wir helfen am Aufbau Deutschlands!“ herausgegeben (Meyer 1939). Sie zeigte einen Stammbaum der wichtigsten aus Knochen herstellbaren Produkte und sollte im Klassenzimmer oder im Schultreppenhaus aufgehängt werden, um die Kleinen dauernd daran 


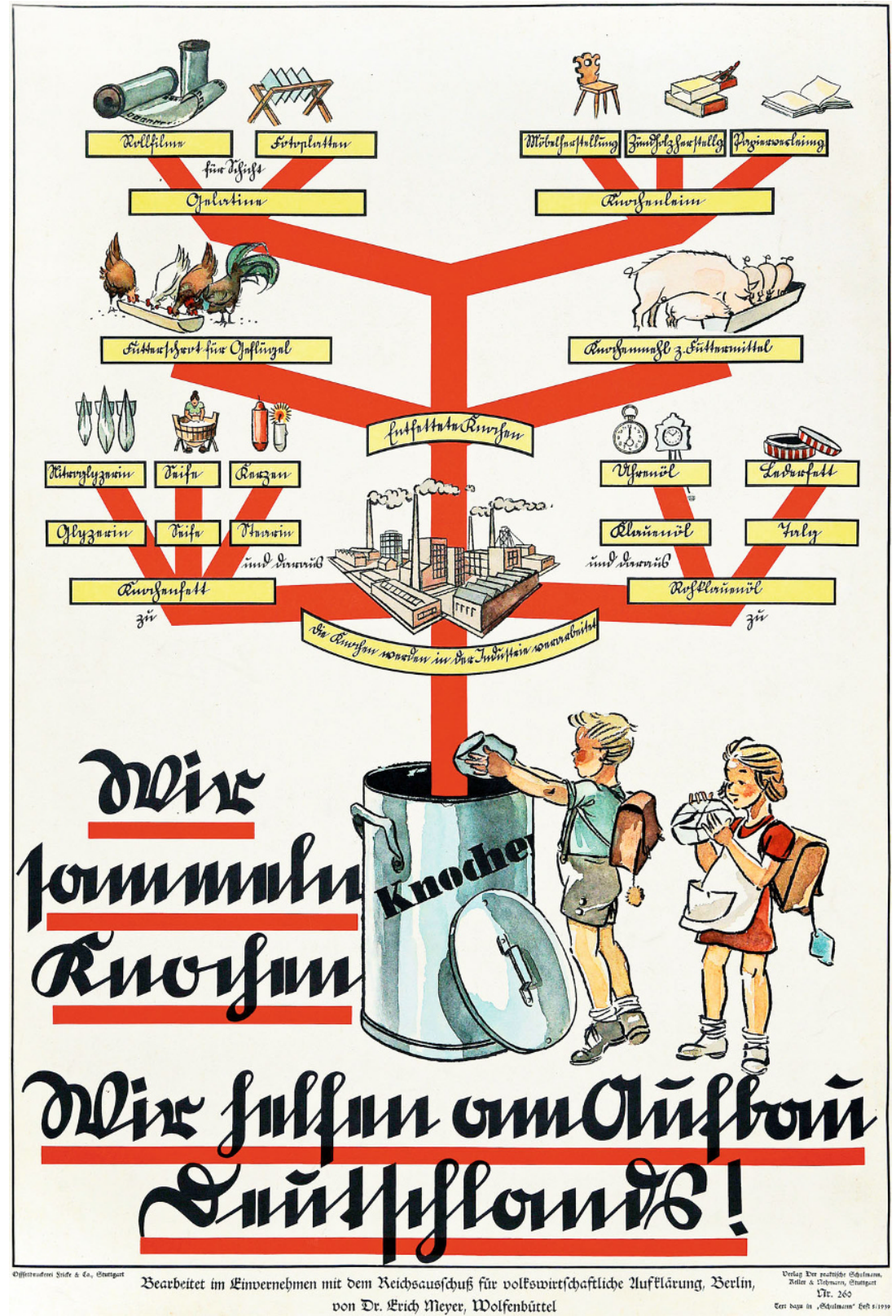

Abb. 2 Im Einvernehmen mit dem Reichsausschuss für volkswirtschaftliche Aufklärung (RVA) konzipierte Lehrtafel zum Thema "Knochenverwertung" für die Volksschule, 1939, Farblithografie auf Papier, $91 \times 63 \mathrm{~cm}$. Schulmuseum Bergisch Gladbach, Sammlung Cüppers, WB-SMP 413. 
zu erinnern, sich angesichts des Krieges „in erhöhtem Maße für die Belange der Knochenverwertung" einzusetzen (Anonym 1940). Zu Füßen des Knochenstammbaums (siehe Abb. 2) war ein mit kurzer Hose bekleideter, blonder Junge dargestellt, der sein Knochenpäckchen gerade in den Sammelbehälter warf. Hinter ihm stand ein blondbezopftes Mädel, das darauf wartete, das Gleiche zu tun.

Die Bildgestaltung suggeriert, dass das Knochensammeln vor allem den Jungen oblag. Ideologisch aufgeladene Texte zum Thema „Wir sammeln Knochen!" fanden sich auch in den Fibeln der ABC-Schützen, etwa in folgendem Lesebuchstück, in dem bezeichnenderweise abermals Jungen die Hauptakteure waren:

Wie stolz marschiert der kleine Fritz mit seinem Ranzen jeden Tag in die Schule! [...] Die Mutter hat ihm einen Beutel mitgegeben. [...] An der nächsten Straßenecke trifft er mit mehreren Schulkameraden zusammen. Auch die tragen Beutel mit Knochen, denn heute ist ja Sammeltag. Wer mag wohl am fleißigsten gesammelt haben? [...] Im Schulhofe steht eine große Tonne. Nun poltern die Knochen in den dunklen Bauch hinein. Auch die anderen Klassen haben tüchtig gesammelt, und morgen wird die Schule dem Händler melden: „Die Tonne ist voll! Sie kann abgeholt werden!“ Wir dürfen stolz sein, daß wir dem Führer durch unser fleißiges Sammeln helfen können (Facharbeitsgemeinschaft deutscher Erzieher 1940: 90).

Auch die vom NS-Lehrerbund (NSLB) herausgegebene, für die Erstbis Viertklässler bestimmte Schülerzeitschrift Deutsche Jugendburg forderte die Kinder mit Versen, Kurzreportagen und einschlägiger Umschlagsgestaltung mehrfach zum Knochensammeln auf. So zeigte ein Titelblatt vom Winter 1941/42 drei Jungen - also abermals nur das männliche Geschlecht - beim Wiegen von Rinderrippen, die allerdings kaum aus Haushaltssammlungen gestammt haben dürften (siehe Abb. 3). Für die Zielgruppe der Erstbis Viertklässler hatte der RVA kurz nach Kriegsbeginn zudem eine farbig illustrierte Broschüre mit dem Titel Auch wir helfen mit! herausgebracht, in der in Versform auf die Kriegswichtigkeit material- und energiesparenden Verhaltens sowie der Altstoff- und Knochensammlungen hingewiesen wurde. ${ }^{13}$

In der ebenfalls vom NSLB herausgegebenen, illustrierten Schülerzeitschrift Hilf mit!, die sich an Fünft- bis Achtklässler wandte, standen Artikel zum Thema Altstoffsammeln zwar nicht im Vordergrund, wurden seit 1937 aber immer wieder eingestreut (Anonym 1937a; Büdingen 1938; Anonym 1940b). Die Schriftleitung der Zeitschrift gab in Zusammenarbeit mit dem Reichskommissar für Altmaterialverwertung nach Kriegsbeginn einen vierseitigen, illustrierten Lehrschaubogen heraus, den die Lehrer mangels 


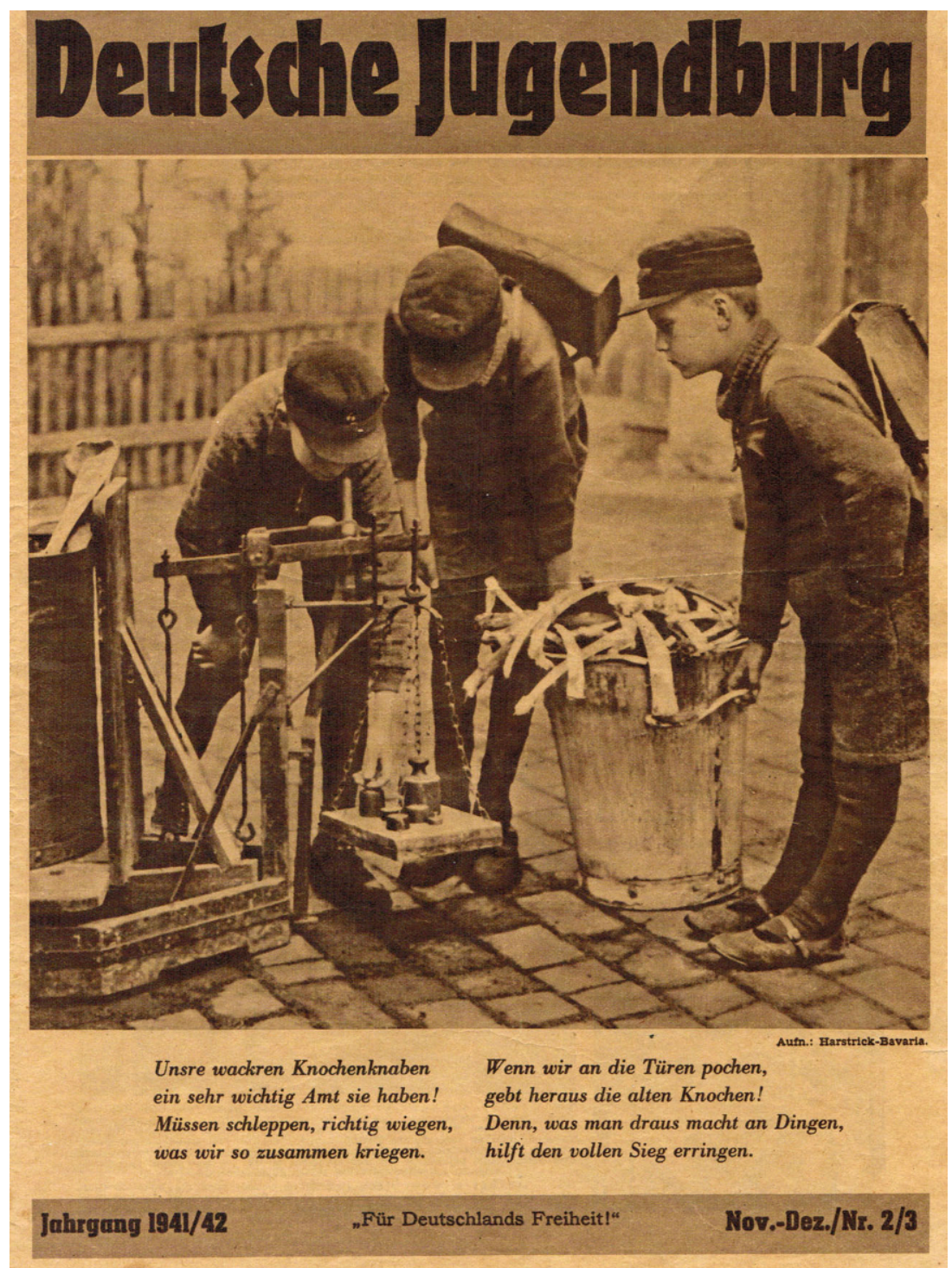

Abb. 3 Titelblatt der vom NS-Lehrerbund herausgegebenen Schülerzeitschrift Deutsche Jugendburg, Winter 1941/42, Schulmuseum Bergisch Gladbach. Das Foto ist eindeutig inszeniert. Da die auffallend uniform gekleideten Jungen nicht in die Kamera schauen, wird der Blick des Betrachters auf inre Tätigkeit und die großen Knochenmengen gelenkt, die die vorbildlichen Schüler zusammengetragen haben. 
geeigneter, nach den neuen Lehrplänen überarbeiteter Schulbücher bei der Thematisierung der Knochenverwertung in der Mittelstufe einsetzen konnten (Reichswaltung [um 1940]). ${ }^{14}$ Geschickt verband er Sachinformation mit politischer Indoktrination, indem er einerseits Fakten über die Verwendungsmöglichkeiten der Knochen lieferte, die Kinder durch Hinweis auf die militärische Bedeutung dieses Rohstoffs aber auch aufforderte, die deutsche Kriegs- und Rüstungswirtschaft mit ihren Sammlungen zu unterstützen. Sogar im Rechenunterricht der Mittelstufe spielte das Thema eine Rolle. In Form eingekleideter Textaufgaben wurde den Schülern Zahlenmaterial über die Knochenverwertung im Deutschen Reich an die Hand gegeben, mit dem sie alltagsbezogene Dreisatzaufgaben lösen mussten (Schiffner 1944: $177 \mathrm{f}$.). ${ }^{15}$ Die politische Vereinnahmung des Rechenunterrichts äußerte sich hier wie in anderen Fällen in der Wahl des als Aufhänger verwendeten Beispiels (Metzner 1931; Dorner 1935). Dem gleichen Zahlenmaterial begegneten die Kinder in Sachbüchern wieder, die auf den vom Reichserziehungsminister publizierten Listen der von den Schulbibliotheken anzuschaffenden Literatur standen (Weber 1999), etwa Ungewitters Verwertung des Wertlosen (Ungewitter 1938: 153) und Lübkes Das deutsche Rohstoffwunder (Lübke 1938: 522). Wie brav die gebetsmühlenartig eingetrommelten Lehrinhalte rezipiert wurden, spiegelt der Aufsatz einer etwa 13-jährigen Volksschülerin zum Thema „Wie ich helfe, den Vierjahresplan zur Durchführung zu bringen“ aus dem Jahr 1941 wieder:

Der Vierjahresplan ist sehr wichtig, denn wir haben keine Kolonien, aus denen wir uns Rohstoffe für unsere Fabriken holen können. Wir haben auch nicht genug ausländisches Geld, um Rohstoffe aus anderen Ländern zu kaufen, darum hat Hermann Göring auf Befehl des Führers den Vierjahresplan geschaffen. Der Vierjahresplan stellt uns die Aufgabe zu sparen. Dieses gilt hauptsächlich für die Hausfrau. Früher, wenn sie alte Knochen hatte, dann wurden sie einfach in den Mülleimer geworfen. Das alles darf heute nicht mehr vorkommen. Jeder Knochen muß gesammelt werden. ${ }^{16}$

\section{Lehrmittel für die Sonderschule}

Es gab sogar ein vereinfachtes Knochenverwertungsschema für die Sonderschule (Anonym 1938). Weil das Verständnis abstrakter Termini bei diesen Schülerinnen und Schülern nicht vorausgesetzt werden konnte, verwendete es nur wenige, anschauliche Begriffe und wies im Gegensatz zur Tafel für die ABC-Schützen nicht auf die militärische Bedeutung der 
Knochensammlungen hin. Die für die Zielgruppe viel zu anspruchsvollen

Fachausdrücke Glycerin und Stearin zeigen aber, dass das Schema wenig durchdacht war. ${ }^{17}$ Obwohl die Sonderschülerinnen und -schüler dem damals propagierten Ideal gesunder, körperlich leistungsfähiger Jugendlicher gewiss nicht entsprachen, wurden sie insofern als „vollwertige“ Volksgenossen betrachtet, als auch von ihnen eine Beteiligung an den schulischen Knochensammlungen erwartet wurde. Das galt besonders für die Kriegsjahre, in denen das Sammeln von Altmaterial als Kriegsdienst der deutschen Schule betrachtet wurde. ${ }^{18}$ Bezeichnenderweise forderte ein Runderlass des Reichserziehungsministers vom Juni 1941 nochmals alle Schulen zum Altstoffsammeln auf (Reichsminister für Wissenschaft, Erziehung und Volksbildung 1941), also auch die Sonderschulen, deren Zöglinge als nützliche Hilfskräfte zur Erledigung einfacher Aufgaben galten (Kremer 2011: 167). Entsprechend dieser Nützlichkeitslogik und im Widerspruch zur damaligen Rassepropaganda mussten auch die ,jüdischen“ Schulen bei den Altstoffsammlungen mitmachen (Anonym 1942h: 114). ${ }^{19}$ Diverse Notizen in der vom NS-Lehrerbund herausgegebenen Zeitschrift Die deutsche Sonderschule belegen, dass die Sonderschülerinnen und -schüler bei den Knochensammlungen durchaus erfolgreich waren und häufig ausgezeichnet wurden. ${ }^{20}$ In ihrer Naivität ließen sie sich wohl ebenso leicht als Müllsammler instrumentalisieren wie die Volksschüler (Anonym 1942b; Anonym 1942c; Anonym 1942e).

\section{Lehrmittel für die Oberschule}

Auf Initiative des Reichskommissars für Altmaterialverwertung war 1938 eine weitere Lehrtafel zum Thema Knochenverwertung erschienen (siehe Abb. 4), die im Gegensatz zu der von Haferkorn und Priemer konzipierten, eher prozessorientierten Tafel (siehe Abb. 1) auf die Zwischen- und Endprodukte abhob und sich daher besonders für den Chemie- und Wirtschaftskundeunterricht eignete. Die Karte wurde mit einem Erläuterungsheft ausgeliefert (Reichskommissar für Altmaterialverwertung 1938a). Ihre Anschaffung wurde den Schulen durch zwei Erlasse des Reichserziehungsministeriums ausdrücklich empfohlen (Reichsminister für Wissenschaft, Erziehung und Volksbildung 1938, 1939). Zusätzlich hatte der Reichskommissar eine aus fünfzig Aufnahmen bestehende Dia-Serie mit dem Titel Der Knochen als Rohstoff und seine Verwertung herausgegeben, ebenfalls mit Begleitheft. Die Kurzinformationen zu jedem Lichtbild ergaben in der Gesamtschau einen kompletten Dia-Vortrag (Reichskommissar für Altmaterialverwertung 1938b). Die Dias illustrierten die technischen Prozesse bei 


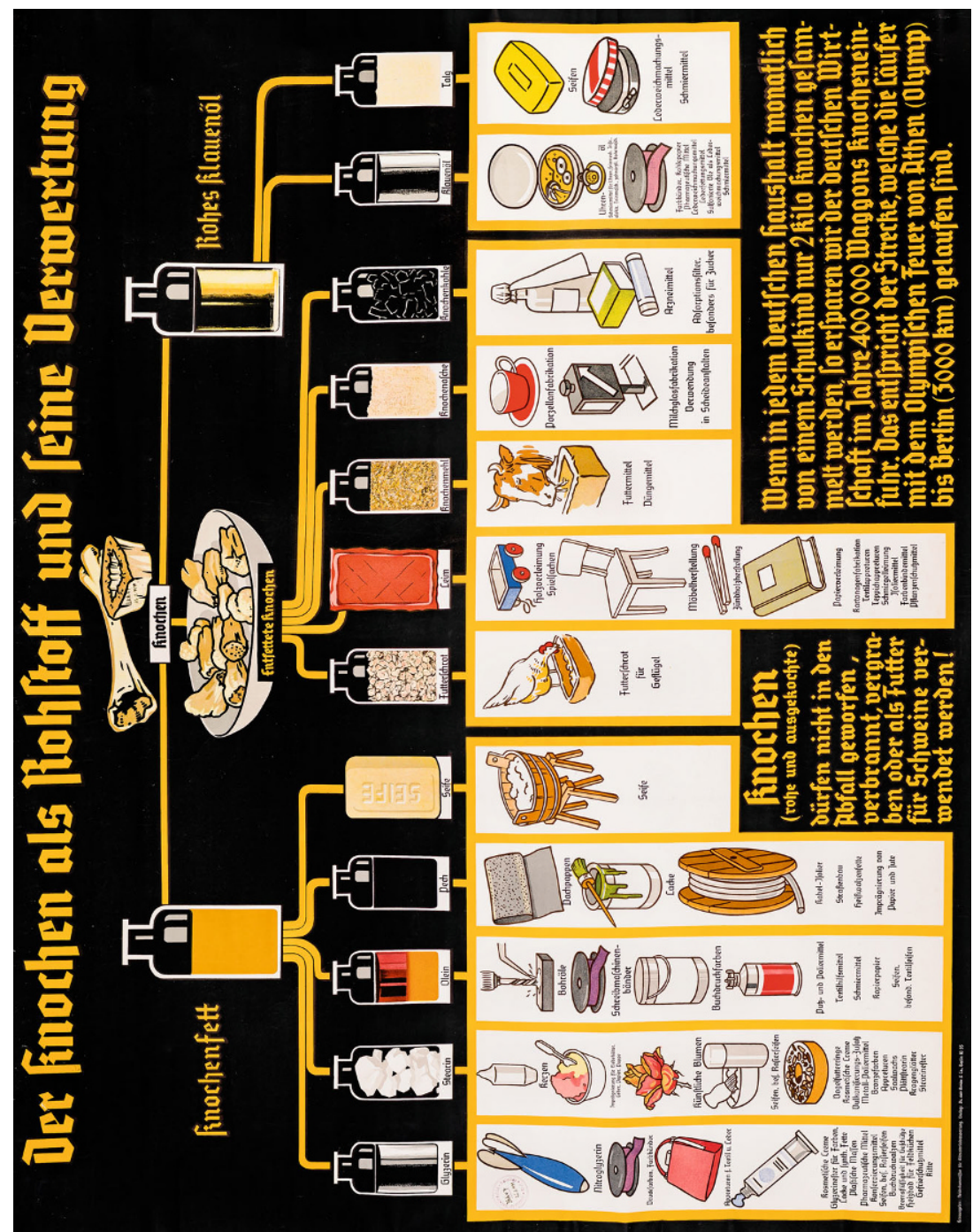

Abb. 4 Vom Reichskommissar für Altmaterialverwertung veröffentlichte Lehrtafel zum Thema „Knochenverwertung" für den Chemie- und Wirtschaftskundeunterricht an Höheren Schulen, 1938, Farblithografie auf Papier, $97 \times 124 \mathrm{~cm}$. Kiel, ZBW - Leibniz Informationszentrum Wirtschaft, $\mathrm{K} 322$.

der Knochenverwertung und vermittelten eine Vorstellung von der Arbeit in wichtigen knochenverarbeitenden Betrieben. Wenn den Schülerinnen und Schülern die eindrucksvollste Form der Veranschaulichung, die Besichtigung eines Schlachthofs beziehungsweise einer Düngemittel-, Leim-, Gelatine-, Kerzen- oder Seifenfabrik nicht geboten werden konnte, ließ sich dieses Manko mit einer Diavorführung kompensieren. ${ }^{21}$ Die Existenz der 
Diaserie zeigt einmal mehr, welche Vielfalt visueller, damals hochmoderner Anschauungsmedien den Lehrern von verschiedenen NS-Institutionen an die Hand gegeben wurde, um den Schülerinnen und Schülern die Relevanz der Knochensammlungen zu verdeutlichen. Weder die Lehrtafel von 1938 noch die Diaserie wurden allerdings ganz neu entwickelt.

Die Art, wie die Größenordnung der im Deutschen Reich verarbeiteten Knochenmengen veranschaulicht wurde, greift klar erkennbar pädagogische Ideen aus dem Film Sammelt Knochen (1918) auf. Einen eigenen, in der NS-Zeit produzierten Unterrichtsfilm zum Thema Knochenverwertung scheint es nicht gegeben zu haben. Wie die 1934 vom Erziehungsministerium gegründete Reichsstelle für den Unterrichtsfilm (RfdU), die 1940 in Reichsanstalt für Film und Bild in Wissenschaft und Unterricht (RWU) umbenannt wurde, zeigt, spielte der Unterrichtsfilm in der Medienpädagogik des „Dritten Reichs“ eine wichtige Rolle (Niethammer 2016). Dank eines 1937 veröffentlichten Bedarfsplans (einer Reaktion auf die per Erlass verfügte Eingliederung des Films in den Schulunterricht) sowie der RWUVerzeichnisse wissen wir, welche Filme damals speziell für den Chemieund Technikunterricht geplant beziehungsweise realisiert worden waren (Weber 1937; Reichsanstalt für Film und Bild in Wissenschaft und Unterricht 1940; Landesbildstelle Württemberg 1943; Kühn 1998: 267-308). ${ }^{22}$ Dass im „Dritten Reich“ kein neuer Film zum Thema Knochenverwertung gedreht wurde, mag daran gelegen haben, dass Hanni Umlauf und ihr Bruder Walter 1937/38 einen solchen zum Oberthema Altstoff - Rohstoff produziert hatten. ${ }^{23}$ Dieser im Auftrag des Reichskommissars für Altmaterialverwertung und der Reichsfrauenführung erstellte Schmalfilm ging auch auf die Knochensammlung und -verwertung ein. Wie sein Vorläufer von 1918 richtete er sich als „volksbildender“ Film in erster Linie an die Hausfrauen. Da er von der Prüfstelle im Februar 1938 ausdrücklich als Lehrfilm zugelassen worden war, durfte er aber auch in der Schule gezeigt werden. ${ }^{24}$

\section{Rohstoff Knochen}

Die Anstrengungen des NS-Regimes, möglichst jeden Abfallknochen im Reich zu erfassen, erfordern eine Erklärung, weshalb dieser Rohstoff eigentlich so begehrt war. Knochen sind aufgrund ihrer besonderen chemischen Zusammensetzung in der Tat ein vielseitig und fast vollständig nutzbares Rohmaterial. Im frischen Zustand bestehen sie zu 50-60 Prozent aus anorganischer Substanz, im Wesentlichen Calciumphosphat, Calciumcarbonat und Magnesiumphosphat (was sie zu einer wertvollen Calcium- und Phos- 
phatquelle machte), und zu etwa 30-40 Prozent aus organischer Substanz, nämlich etwa 35 Prozent Gerüsteiweißen und 5-15 Prozent Fett (was sie zu einem Protein- und in geringerem Umfang auch zu einem Fettlieferanten machte). Der Rest ist Wasser. Das Verhältnis der einzelnen Bestandteile zueinander schwankt je nach Alter, Rasse, Ernährungszustand und dem Körperteil des Tieres, aus dem die Knochen stammen, erheblich, sogar bei derselben Tierart (Gerngross \& Goebel 1933: 171-176; Ostertag et al. 1958: 364-366).

Als eine der ersten Branchen der chemischen Industrie, die Abfälle als Rohstoff verwendete, war Anfang des 19. Jahrhunderts die knochenverarbeitende Industrie entstanden (Fleck 1878: 6-84). 1857 folgte die Teerfarbenindustrie, die den Steinkohlenteer, das Abfallprodukt der Kokereien und Leuchtgasfabriken, nutzte. Die phosphatreichen Knochen wurden zunächst ausschließlich zu Düngemitteln verarbeitet, wobei die Entwicklung dieses Fabrikationszweiges zunächst vor allem von Großbritannien ausging (Wagner 1868: 607-616; 621-623). Da das inländische Rohmaterial den Bedarf bald nicht mehr deckte, begannen die Briten, Knochen aus aller Welt zu importieren. In den 1820er Jahren sollen sie sogar die Schlachtfelder der gegen Napoleon geführten Befreiungskriege nach Knochen durchwühlt und diese nach Großbritannien verschifft haben (Liebig 1861: 7; Vogel 1874: 45f.). Der Bedarf an Phosphatdüngern stieg deutlich an, nachdem Liebig darauf hingewiesen hatte, dass Phosphor, Stickstoff und Kalium für die Pflanzenernährung unverzichtbar sind und dem Boden nach der Ernte zur Aufrechterhaltung seiner Fruchtbarkeit wieder zugeführt werden müssen. Um die große Nachfrage zu decken, wurden seit den 1860er Jahren neben Knochen auch phosphathaltige Erze zu Düngern verarbeitet. Diese Mineralien mussten jedoch importiert werden (Ost 1942: 385-396; Welsch 1981: 39f.). In den 1880er Jahren wurde entdeckt, dass sich die bei der Stahlherstellung anfallende Thomas-Schlacke in vermahlener Form ebenfalls als Phosphatdünger nutzen ließ. Trotz seiner umfangreichen Stahlproduktion war das Deutsche Reich allerdings auch bei diesem Rohstoff, einem klassischen Industrieabfallprodukt, nicht autark (Welsch 1981: 79; $127 \mathrm{f}$.). Als die deutsche Düngemittelindustrie im Ersten Weltkrieg vom Import ausländischer Phosphaterze abgeschnitten wurde, begann sie sich auf die Nutzung heimischer Ressourcen zu besinnen: die mageren, bis dahin als nicht aufbereitungswürdig betrachteten Lahn-Phosphate sowie das seit Anfang des 20. Jahrhunderts bereits weitgehend durch die Mineraldünger verdrängte Knochenmehl. Wohlwissend, dass das Deutsche Reich dank des Haber-Bosch-Verfahrens bei der Herstellung von Nitratdüngern autark, bei der Produktion von Phosphatdüngern aber partiell auf ausländische Rohstoffe angewiesen war, wurden die im Ersten Weltkrieg verfolgten Ausweichstrategien im „Dritten Reich“ reaktiviert: Um im Kriegsfall mehr 
heimische Knochen zu Phosphatdüngern (und anderen dringend benötigten Knochenverwertungsprodukten) verarbeiten zu können, schuf man mit den 1937 initiierten schulischen Knochensammlungen rechtzeitig vor dem Auftreten einer konkreten Krisen- und Mangelsituation die Infrastruktur, die zur Erfassung dieses wichtigen Industrierohstoffs erforderlich war.

Seit etwa 1848 wurden die Knochen entfettet, bevor sie zu Düngemitteln vermahlen wurden. Dadurch verbesserte sich nicht nur die Düngewirkung; die Entfettung war auch lukrativ. Das bei der Spaltung des Knochenfetts entstehende Fettsäuregemisch ließ sich nämlich zu billigen Seifen (Kern-, Schmier-, Textilseifen) ${ }^{25}$ oder zu Stearinkerzen verarbeiten; zudem fiel bei der Fettspaltung Glycerin an, ein Schlüsselprodukt der chemischen Technik. Es wurde und wird bei der Herstellung von Salben, Zahncremes und Kosmetika sowie als Textilappretur verwendet. Wegen seiner Zähflüssigkeit diente und dient es als Bremsflüssigkeit (auch in Geschützen), im Gemisch mit Wasser als Frostschutzmittel (auch in Wehrmachtsfahrzeugen) sowie als Weichmacher für Filme, Farbbänder, Kopiertinten und Druckfarben. Den größten Glycerinbedarf hatte seit der Erfindung des Dynamits (Nitroglycerin) aber die kriegswichtige Sprengstoffindustrie. Gleichzeitig mit der Fettgewinnung begannen die Knochenmehlfabrikanten, einen Teil der entfetteten Knochen durch Verkohlen unter Luftabschluss zu Knochenkohle zu verarbeiten. Sie wurde als Schwarzpigment verwendet, etwa zur Herstellung von Schuhwichse und Malerfarben, aber auch als medizinische Kohle; große Bedeutung hatte sie zudem als Adsorptionsmittel für industrielle Zwecke. ${ }^{26}$

Ein weiterer Meilenstein in der Geschichte der Knochenverwertung war erreicht, als Anfang des 19. Jahrhunderts zunächst in Frankreich und seit den 1860er Jahren auch in Deutschland die ersten Fabriken entstanden, in denen die entfetteten Knochen zu Leim und Gelatine verarbeitet wurden. In beiden Fällen handelte es sich um Umwandlungsprodukte der in den Knochen enthaltenen Gerüsteiweiße, die unter Druck mit heißem Wasser gespalten wurden. Der Prozess wurde so gelenkt, dass bei der Knochenleimherstellung ein gut klebendes und bei der Gelatineherstellung ein gut gelierendes Produkt entstand (Bergmann 1919). Dank der in der zweiten Hälfte des 19. Jahrhunderts zunehmenden Bedeutung der Fotografie sowie des Aufstiegs der fotografischen Industrie entwickelte sich die Gelatinefabrikation gerade im Deutschen Reich zu einem wichtigen Industriezweig. Seit Gründung des Agfa-Werkes in Wolfen besaß es die größte Filmfabrik Europas und nach der Eastman-Kodak Company in den USA sogar die zweitgrößte der Welt. An die Qualität der Fotogelatine wurden extrem hohe Anforderungen gestellt (Baier 1977: 261-280). Nur ein Produkt, das aus fettarmen, frischen Schlachthausknochen hergestellt worden war, garantierte haltbare, fleckenfreie Fotos und Filme. ${ }^{27}$ In einer Kriegssituation 
war die Verfügbarkeit von genügend Filmmaterial und Fotopapier, deren Herstellung in beiden Fällen Fotogelatine erforderte, für Dokumentationsund Propagandazwecke unverzichtbar. Das galt auch für andere technisch relevante Gelatinesorten, die beispielsweise als Appreturmittel oder Anlaufschutz für Gasmaskengläser verwendet wurden. Gleichermaßen wichtig war die Speisegelatine, die die Pharmaindustrie zum Verkapseln von Medikamenten benötigte; sie wurde aber auch zu Puddingpulver verarbeitet und von der Fisch- und Fleischkonservenindustrie als Formgeber verwendet.

Zusammenfassend lässt sich sagen, dass Knochen im 19. Jahrhundert also zur Rohstoffbasis mehrerer Branchen der chemischen Industrie geworden waren (Stohmann \& Engler 1872: 386-398; Stohmann \& Engler 1874: 652-674). Die Nachfrage nach diesem außerordentlich vielseitig nutzbaren Industrierohstoff war schon in den späten 1870er Jahren derart gestiegen, dass der Bedarf auch im Deutschen Reich nicht mehr aus heimischen Ressourcen, sondern nur noch durch zusätzliche Knochenimporte aus Südamerika und Indien gedeckt werden konnte.

\section{Kritische Analyse der Schulwandbilder}

Im Begleitheft zur Lehrtafel des Reichskommissars von 1938 wird behauptet, dass in der NS-Zeit zwischen achtzig und hundert verschiedene Produkte aus Substanzen hergestellt wurden, die bei der Knochenverwertung anfielen (Reichskommissar für Altmaterialverwertung 1938a). Die Tafel zeigte, dass aus Knochen nicht nur technisch, landwirtschaftlich oder militärisch wichtige Produkte hergestellt wurden (Bohröle, Kitte, Lacke, Druckfarben, Isolier-, Appretur-, Dünge-, Futter- und Pflanzenschutzmittel, Glycerin, Nitroglycerin), sondern auch Gebrauchsgegenstände (Kerzen, Schreibmaschinenbänder, Schuhwichse, Kopierpapier, Zündhölzer, Möbelleim, Arzneimittel, Rasier- und Hautcremes) und Luxuswaren (Kunstblumen, Knochenporzellan). Die Lehrtafel, eine immer wieder reproduzierte, auch als Ausstellungsexponat verwendete Bildikone, ${ }^{28}$ vermittelte die Botschaft, dass die Knochensammlungen in vielerlei Weise da$\mathrm{zu}$ beitrugen, den Lebensstandard und das Konsumniveau des deutschen Volkes zu sichern oder gar zu heben.

Vermutlich dürfte den wenigsten Betrachtern aufgefallen sein, dass die Herstellung von Kunstblumen, Kerzen, Milchglas, Bohnerwachs oder Vogelfutterringen wirtschaftlich eine weitaus geringere Rolle spielte als die in mehreren Zweigen des Produktestammbaums angeführte Seifenherstellung und die Glyceringewinnung (Schlenker 1932: 206-253). Im Bestreben, 
möglichst viele Alltagsartikel aufzuzählen, deren Herstellung direkt oder indirekt von der Verfügbarkeit des Rohstoffs Knochen abhing, wurden auf der Schulwandtafel des Reichskommissars zahlreiche Produkte aufgeführt, die hinsichtlich ihrer wirtschaftlichen Bedeutung nicht gleichwertig und daher nicht miteinander vergleichbar waren. Des Weiteren fällt auf, dass die Darstellung völlig ahistorisch und bezogen auf die Gegebenheiten im "Dritten Reich“ teils sogar falsch war. So hatte das im 19. Jahrhundert wichtige Düngemittel Knochenmehl seine Bedeutung in den 1930er Jahren längst zugunsten der Mineraldünger eingebüßt. Andererseits wurde die Herstellung der Fotogelatine, die bis heute nur aus Knochen gewonnen werden kann, erstaunlicherweise nicht erwähnt, obwohl sie militärisch besonders wichtig war. Auf den für die Berufs- beziehungsweise die Volksschule konzipierten Lehrtafeln wurden zwar Rollfilme abgebildet, allerdings auch die in den 1930er Jahren bereits ungebräuchlichen Gelatinetrockenplatten. Die Inkonsistenzen spiegeln die polykratischen Strukturen des NS-Regimes wieder und lassen vermuten, dass sich die verschiedenen Institutionen, die die Lehrmaterialien erarbeitet hatten, nicht untereinander abgestimmt hatten. Statt zwischen wirtschaftlich wichtigen Haupt- und unbedeutenden Randprodukten zu differenzieren sowie obsolet gewordene Knochenverwertungsprodukte kenntlich zu machen und sich nur auf die im „Dritten Reich“ bedeutsamen Nutzungen zu beziehen, ging es dem Reichskommissar vor allem darum, möglichst viele prinzipiell aus Knochen herstellbare Produkte auf seiner Tafel aufzuführen. Dabei wurde die Information unterschlagen, dass manche nur aus frischen Schlachthausknochen gewonnen werden konnten, nicht jedoch aus den Sammelknochen, die die Schüler zusammentrugen. Bezeichnend ist ferner, dass keine der hier vorgestellten Lehrtafeln die Speisefettherstellung aus Knochen erwähnte, obwohl sie seit 1936 erlaubt war. ${ }^{29}$ Vermutlich war dieses heikle Thema bewusst ausgeklammert worden, weil es ungute Erinnerungen an die staatlich konzessionierte Absenkung des Lebensmittelstandards im Ersten Weltkrieg geweckt hätte (Keller 2015: 26). Damals war tatsächlich Knochenfettmargarine auf den Markt gekommen (Pelzer \& Reith 2001: 54), die im Volk „gewisse Vorurteile gegen die Knochen“ hervorgerufen hatte (Ziegelmayer 1936: 135). Gleichermaßen fällt auf, dass die Speisegelatine nicht erwähnt wurde, obwohl die Abbildung eines Wackelpuddings oder von Fruchtgummis sicher kindgerecht gewesen wäre. Die ekelerregende Vorstellung, dass die Sammelknochen zu Nahrungsmitteln verarbeitet werden könnten, sollte offensichtlich gar nicht erst aufkommen. Durch solche und ähnliche Vereinfachungen, Beschönigungen, Auslassungen und Falschdarstellungen entlarven sich die im „Dritten Reich“ produzierten Lehrtafeln als Propagandamittel. 
Wegen der besonderen wirtschaftlichen Bedeutung der aus Knochenfett herstellbaren Seife und des bei der Fettspaltung anfallenden Glycerins war die Entfettung der Knochen bis auf maximal ein Prozent Restgehalt im „Dritten Reich“ seit 1936 gesetzlich vorgeschrieben (Anonym 1942a). ${ }^{30}$ Diese Bestimmung zeigt, dass es dem NS-Staat bei den Knochensammlungen in erster Linie darum ging, den eklatanten Fettmangel zu lindern und in den Besitz der für die Seifenherstellung unverzichtbaren Fettsäuren sowie des militärisch wichtigen Glycerins zu gelangen. ${ }^{31}$ Dabei kam dem NS-Staat zupass, dass sich die entfetteten Knochen anschließend noch zu Leim sowie den ebenfalls dringend benötigten Dünge- und Futtermitteln weiterverarbeiten ließen. Da das Alter der Knochen für ihre Verwendungsmöglichkeiten entscheidend ist - durch Licht-, Sauerstoff- und Bakterieneinwirkung unterliegt gerade ihr ohnehin sehr geringer Fettanteil schnell wertmindernden chemischen Veränderungen - konnte das bei Schulsammlungen akquirierte Material nur zu bestimmten Produkten verarbeitet werden; zur Herstellung von Fotogelatine oder Speisefett war es ungeeignet. Während des Krieges waren die Güter, die aus den qualitativ minderwertigen Sammelknochen hergestellt werden konnten, trotz ihrer Mängel aber unverzichtbar geworden. Während der konkrete ökonomische Nutzen der schulischen Knochensammlungen noch zu klären wäre, liegt ihr ideologischer Wert auf der Hand (Köstering 1997: 49; Weber 2013a). Zweifellos dienten sie der politischen Erziehung zu sparsamem Umgang mit knappen Rohstoffen und vollem Einsatz für die Volksgemeinschaft. Sie trugen dazu bei, die Heimatfront zu mobilisieren und schon den Kindern ein Bewusstsein für die Bedeutung scheinbar wertloser Abfallstoffe zu vermitteln. Dabei kam der Schule eine Schlüsselrolle zu. Die ideologische Funktion der Knochensammlungen erklärt auch, warum die hier vorgestellten Unterrichts- und Anschauungsmaterialien im Detail unausgewogen, ungenau, widersprüchlich und teils sogar fehlerhaft waren: Letztlich waren sie von den NS-Instanzen, die an ihrer Erarbeitung beteiligt und entweder dem Reichserziehungs-, dem Reichspropaganda- oder dem Reichswirtschaftsministerium unterstellt waren, in erster Linie als Propaganda- und nicht als seriös und ausgewogen informierende Lehrmittel konzipiert worden. Dass die Begleitbroschüre zur Lehrtafel des Reichskommissars ausgerechnet von Egon Witthuhn, dem Beauftragten für Altmaterialerfassung der NSDAP im Gau Berlin, verfasst und mit einer Widmung von Hermann Göring versehen worden war, bestätigt diesen Eindruck (Reichskommissar für Altmaterialverwertung 1938a). ${ }^{32}$ 


\section{Fazit}

Altstoffe, darunter Knochen, wurden in den Kriegsjahren auch außerhalb des Deutschen Reichs gesammelt und verwertet. Auch dort gab es eigens produzierte Unterrichtsmittel, um die Altstoffverwertung in der Schule zu behandeln. Bislang ungeklärt ist die Frage, ob die Knochenverwertung in diesen Ländern eine ebenso prominente Rolle wie im Deutschen Reich spielte und ob dort eine ähnliche Vielfalt verschiedener, nach Zielgruppen differenzierter Lehrmaterialien eingesetzt wurde. Für die DDR ist die Kontinuität der Altstoff- und Knochensammlungen belegt. In der Tat wurde in der jungen DDR eine Lehrtafel produziert, die die Schülerinnen und Schüler zwecks „Erfüllung des Fünfjahresplanes“ zum Knochensammeln aufforderte; die Ikonografie dieses Schaubildes orientierte sich unverkennbar an der des Reichskommissars von 1938 (siehe Abb. 4). Im Falle des kriegswichtigen Glycerins fällt allerdings eine deutliche „Entmilitarisierung“ der Inhalte auf: Statt der Bombe, die auf der Tafel des Reichskommissars von 1938 dargestellt wurde, ist auf dem Schulwandbild aus der DDR eine Zinntube für Haut- und Rasiercremes zu sehen. Es wurde also nur noch auf die zivilen Nutzungsmöglichkeiten der klassischen Dual-use-Substanz Glycerin hingewiesen, während die militärischen Anwendungen gar nicht mehr angesprochen wurden. ${ }^{33}$

Dass der Knochenverwertung im Schulunterricht des „Dritten Reichs“ eine solch auffallende Sonderstellung zukam, lässt sich erklären: Im Gegensatz zu allen anderen damals gesammelten Altstoffen ließ sich nur das Rohmaterial Knochen zu einem derart vielfältigen Produktespektrum verarbeiten. Nicht zufällig mussten die deutschen Schüler als ersten Altstoff überhaupt ausgerechnet Knochen sammeln, für die ihnen nach Einführung des Prämiensystems zudem mehr Punkte als für alle anderen Wertstoffe (Lumpen ausgenommen) gutgeschrieben wurden. Knochen entsprachen dem in der NS-Zeit propagierten Ideal des „total“ verwertbaren Altstoffs besonders gut; die fast restlose Verwertbarkeit war vor allem dann gegeben, wenn bei ihrer Aufarbeitung weitgehend auf gewisse, stets mit Materialverlusten einhergehende Reinigungsschritte verzichtet wurde. Ein Alleinstellungsmerkmal der Knochen war zweifellos, dass sie im Gegensatz zu allen anderen Altmaterialien nutzbare Mengen anorganischer und organischer Inhaltsstoffe enthielten. Der Rohstoff Knochen war deshalb bestens geeignet, um den Schülern an einem einzigen Fallbeispiel aufzuzeigen, wie sich diese beiden chemisch so verschiedenen Stoffgruppen nacheinander gewinnen und nutzen ließen - die Schulversuche machten gerade diesen Aspekt besonders deutlich. Die von den Schülern zusammengetragenen Sammelknochen besaßen zweifellos nicht die Qualität frischer Schlachthausknochen, sie unterschieden sich aber nur geringfügig von der Ware, 
die das Deutsche Reich aus Südamerika und Indien importierte. Im Gegensatz zu Lumpen, Altpapier oder Altmetall, die als industriell hergestellte Fertigfabrikate wirklich recycelt werden mussten, um die bei ihrer Produktion eingesetzten Rohstoffe wiederzugewinnen (Petzold 1941), waren Sammelknochen im Prinzip ein (weitgehend) unbearbeiteter Primärrohstoff ihre vorherige Nutzung in der Küche veränderte weder ihren anatomischen Aufbau noch ihren Mineralgehalt; auch ihr Fett- und Proteingehalt reduzierte sich durch „normales“ Kochen und Braten (also kein stundenlanges Erhitzen, keine Druckanwendung im Schnellkochtopf) nur um höchstens ein Drittel.

Da die Requirierung und Verwertung von Altstoffen immer auf die Wirtschafts- und Industriestruktur des jeweiligen Landes optimiert war und auf diese zurückverwies, besaßen die verschiedenen Altmaterialien nicht überall die gleiche Bedeutung wie im Deutschen Reich: ein Land mit reichen Eisen- oder Buntmetallvorkommen war natürlich weniger auf die Sammlung von Schrott oder anderen Altmetallen angewiesen als ein metallarmes Land; ein waldreiches musste weniger Altpapier recyceln als ein holzarmes; eines, das, wie das Deutsche Reich, über eine Kautschuksynthese verfügte, konnte Altgummisammlungen eine niedrigere Priorität zugestehen als eines, das dieses Know-how nicht besaß. Es wäre daher zu prüfen, ob die deutsche Wirtschaft in Anbetracht der lange vor dem Zweiten Weltkrieg konstatierten „Fettlücke“ (Reith 2002) möglicherweise einen größeren Fettbedarf hatte als die ausländische Industrie, so dass der Knochenrequirierung in der deutschen Wertstoffpropaganda auch deshalb ein höherer Stellenwert zukam als andernorts. In diesem Kontext ist bezeichnend, dass der Reichskommissar für Altmaterialverwertung versuchte, in Analogie zum bekannten Teerstammbaum einen reich verzweigten, viele „Produkt-Früchte“ tragenden Knochenstammbaum zu konstruieren (Kühn 1939: 15; Weber 2014: 164f.), dessen tragende Äste vor allem die Bedeutung der Knochen für die Fettgewinnung visualisierten. Angesichts des geringen Fettgehaltes von Knochen stellte diese Darstellung die tatsächlichen Gegebenheiten geradezu auf den Kopf und entlarvte sich dadurch als reine Propaganda. Diese nur für das Deutsche Reich belegte Darstellung lässt erkennen, ${ }^{34}$ dass dem Rohstoff Knochen eine ähnliche Schlüsselrolle wie dem Teer zugeschrieben wurde. Beide Stammbäume suggerierten, dass sich die deutsche (Kriegs-)Wirtschaft mit den heimischen Rohstoffen Knochen und Teer, klassischen Abfallstoffen, auf zwei starke, die Verfügbarkeit eines üppigen Warenangebots sichernde Pfeiler stützen konnte (Anonym 1938a: 98f.). Beide Bildikonen visualisierten den durch Ungewitters Buch popularisierten Slogan von der Verwertung des Wertlosen; sie verdeutlichten, dass die deutsche chemische Industrie die Autarkiebemühungen des Reichs unterstützte, indem sie vermeintlich wertlose Edukte durch chemi- 
sche Prozesse in eine Fülle wertvoller Produkte verwandelte. Der Rohstoff Knochen war also aus mehreren Gründen ideal, um die NS-Wertstoffpolitik und die Devise „Altstoff ist Rohstoff“ in jener Institution zu propagieren, der bei der Sammlung gerade dieses Materials eine so zentrale Funktion zukam: der Schule.

\title{
Archive, Sammlungen, Museen
}

\author{
Bergisch Gladbach, Schulmuseum \\ Berlin, Bundesarchiv-Filmarchiv \\ Berlin, Deutsches Historisches Museum \\ Frankfurt, Deutsches Filminstitut \\ Freiburg im Breisgau, Staatsarchiv \\ Kiel, ZBW - Leibniz-Informationszentrum Wirtschaft \\ München, Institut für Zeitgeschichte \\ Würzburg, Forschungsstelle Historische Bildmedien der Universität
}

Open Access Dieser Artikel wird unter der Creative Commons Namensnennung 4.0 International Lizenz (http://creativecommons.org/licenses/by/4.0/deed.de) veröffentlicht, welche die Nutzung, Vervielfältigung, Bearbeitung, Verbreitung und Wiedergabe in jeglichem Medium und Format erlaubt, sofern Sie den/die ursprünglichen Autor(en) und die Quelle ordnungsgemäß nennen, einen Link zur Creative Commons Lizenz beifügen und angeben, ob Änderungen vorgenommen wurden.

\section{Anmerkungen}

1 Die Forschungsstelle NS-Pädagogik an der Goethe-Universität Frankfurt am Main hat auf https://web.archive.org/web/20180316074650/https://forschungsstelle.files. wordpress.com/2017/11/06_09_2017-bibliographie-bibliothek-fs-katalog.pdf (S. 130134) Literatur zum Thema „Erziehung und Schule“ zusammengestellt (Link überprüft am 09.04.2018).

2 Für die Schule angefertigte Großbilder zur Veranschaulichung von Sachzusammenhängen sind seit dem 18. Jahrhundert bekannt. Erste Bilderserien kamen seit 1830 auf den Markt, 1850 begann die umfangreichere Produktion. Einige Verlage spezialisierten sich auf die Produktion von Lehrtafeln, die es für alle Schularten und Fächer gab. Mit der Abkehr vom Frontalunterricht und der Einführung von Overhead-Projektion und Powerpoint-Präsentation verloren die Schulwandbilder ihre einstige Bedeutung. Heute sind sie weitgehend aus dem Unterricht verschwunden (Müller 1997).

3 Deutsches Reich (Köstering 1997, 2003; Berg 2015; Weber 2013a, b, 2019; Weber \& Denton 2018); Italien (Alimenti 1940: 35, 54); Kroatien (Anonym 1943b); Slowakei (Anonym 1942i); Protektorat Böhmen und Mähren (Anonym 1940a); Niederlande (Oldenziel \& Veenis 2001: 98); Frankreich (Denton 2013, 2014, 2016); Estland (Anonym 1942f); Sowjetunion (Müller 1991: 290-292); USA (Zimring 2009; Strasser 2013; Kim- 
ble 2014; Braun 2014); Kanada (Durflinger 2006: 87; Perrun 2009; Mosby 2014); Großbritannien (Thorsheim 2013; 2015; Irving 2016; Bullus 2017); Schweiz (Anonym 1944; Rothenbach-Abderhalden 1948: 21; Anonym 1946); Lettland (Anonym 1942d).

$4 \quad$ Kinttof (1935); Morgner (1935); Staufenbiel (1936); Pricks (1937); Schulze (1938); Wüstner (1938); Grüntzig (1939), Scharf \& Golombeck (1940).

5 Der Versuchsablauf gestaltete sich folgendermaßen: Nach dem Nachweis der anorganischen Inhaltsstoffe wurden die Knochen entkalkt, entfettet und aus dem Fett eine Seife hergestellt. Ein Teil der entfetteten Knochen wurde dann zu Knochenkohle, der andere zu Leim beziehungsweise Gelatine aufgearbeitet. Mit letzterer wurde eine fotografische Platte hergestellt.

6 Filmarchiv des Bundesarchivs, Berlin, 22969, https://web.archive.org/web/2018040914 3027/https://www.filmothek.bundesarchiv.de/video/58908 (Link überprüft am 09.04. 2018). Der Film wurde mit Unterstützung der Firma Scheidemandel in Berlin gedreht, dem wichtigsten Knochenverwerter im Reich (Das Spezial-Archiv der deutschen Wirtschaft 1931: 109-114).

7 ESM, Abb. 5: Vom Reichskommissar für Altmaterialverwertung und dem Reichsbeauftragten der NSDAP für Altmaterialerfassung herausgegebener, als Werbegeschenk verteilter Wandkalender für das Jahr 1943, Schulmuseum Bergisch Gladbach.

81942 wurde die Dienststelle eines „Sonderbeauftragten für Knochen und Horn“ geschaffen (Anonym 1942g).

91943 stellte das Reich eine Million Bleistifte als Prämie zur Verfügung (Anonym 1943).

10 https://web.archive.org/web/20170828073452/https://forum.axishistory.com/down load/file.php?id=294460\&sid=dba49cbf9302e8e86b788e8d72004644\&mode=view (Link überprüft am 09.04.2018).

11 ESM, Abb. 6: Bogen zum Einkleben der für ein Pfund/ein Kilo Sammelknochen vergebenen Marken; vollständig beklebt berechtigte er zum Kauf eines Stückes Kernseife, 1944, Schulmuseum Bergisch Gladbach; ESM, Abb. 7: Werbeplakat „Sammelt Knochen“ für das Bezugsscheinsystem „Ein Stück Seife für fünf Kilo Knochen“, 1944, Farblithografie auf Papier, $30 \times 21,5 \mathrm{~cm}$, Institut für Stadtgeschichte, Frankfurt am Main, Materialsammlung Lerch, S6b-93-A, 51 (Altstoffsammlung). Das Plakat ist ein Werk des Grafikers Oswald Schiffers, der zeitweise für das Reichspropagandaministerium arbeitete.

12 Vgl. Akte „Rattenplage an der Knochen- und Altwarensammelstelle von Max Deutsch“, 1938/39, Staatsarchiv Freiburg, B 728/1 Nr. 6912. Die bis 01.04.1938 in „jüdischem“ Besitz befindliche Rohproduktenhandlung Max Deutsch in Offenburg wurde nach ihrer „Arisierung“ von einem deutschen Altwarenhändler weitergeführt. Wie der Vorinhaber betrieb er eine Altmaterial- und Knochensammelstelle in einem Wohngebiet, die unerträglich stank und Ratten, Mäuse und Fliegen anzog. Nach massiven Beschwerden der Anwohner musste das Knochenlager zum 01.09.1938 ins Industriegebiet außerhalb der Stadt verlegt werden.

13 ESM, Abb. 8: Titelblatt und Seite 14 aus der vom Reichsausschuss für volkswirtschaftliche Aufklärung (RVA) herausgegebenen Broschüre „Auch wir helfen mit!“, Leporello, ca. 1940, Schulmuseum Bergisch Gladbach. Der RVA war unter anderem für die Aktionen „Kampf dem Verderb“ und „Kohlenklau“ verantwortlich (Reith 2010).

14 ESM, Abb. 9: Vom NS-Lehrerbund konzipierter „Lehrschau-Bogen“ zum Rohstoff Knochen, um 1940, doppelseitig bedruckt, DIN A3, Institut für Zeitgeschichte, München, Archiv Z_1482_0018.

15 ESM, Abb. 10: Textaufgabe aus dem Kapitel „Kampf dem Verderb!“ eines Volksschulrechenbuchs (Schiffner 1944: 177 f.). Ähnlich aufgebaute Rechenaufgaben wurden auch in Anzeigeform veröffentlicht (Reith 2010: 153, dort Abb. 9).

16 Aufsatz Nr. 8 von Maria Krey, 7. Volksschulklasse, Teltow 10.01.1941, Transkription Peter Joerißen, Schulmuseum Bergisch Gladbach, ARCH 43.

17 Der Kurzfilm Sammelt Knochen von 1918 zeigte beispielsweise die militärische Bedeutung des Knochensammelns zur Gewinnung von Fetten, die für die Seifenherstel- 
lung benötigt wurden, sowie die Gewinnung des kriegswichtigen Glycerins. Vgl. auch Anm. 31.

18 Es wurde aber vorausgesetzt, dass die Sammlungen den Unterricht nicht beeinträchtigten.

19 Seit 1943 mussten jedoch zumindest die Berufs-, Berufsfach- und Fachschulen im Regierungsbezirk Kattowitz nicht mehr an den Altstoffsammlungen teilnehmen (Anonym 1943c).

20 Die Sonderschullehrer wollten durch gute Sammelergebnisse die wiederholt in Frage gestellte Existenzberechtigung der "Hilfsschule" beweisen und zeigen, dass auch ihre Schüler für „Führer“ und Volksgemeinschaft nützlich sein konnten (Neubert 1940, 1941).

21 Vorrang gegenüber dem Dia oder Film hatte aber die Betriebsbesichtigung (Erziehung und Unterricht in der Höheren Schule 1938: 167).

22 Für den Chemieunterricht stellte die RWU vor allem Filme zur Verfügung, die die Verarbeitungsmöglichkeiten vierjahresplanrelevanter Rohstoffe wie Glas, Braunkohle, Steinkohle, Erze, Stahl, Ton, Kalk, Karbid, Zucker und Textilfasern thematisierten (Landesbildstelle Württemberg 1943: 12f.).

23 Filmarchiv des Bundesarchivs, Berlin, 2811. In den besetzten Niederlanden wurde der Film „Wie't kleine niet eert" („Wer die kleinen Dinge nicht ehrt") produziert und seit 1942 vorgeführt (Oldenziel \& Veenis 2001: 96). Das britische „Ministry of Information“ produzierte 1944 den Zeichentrickfilm „Bones, Bones, Bones - Save Bones“, vgl. https://web.archive.org/web/20180409142912/https://www.britishpathe.com/video/ bones-bones-bones-save-bones (Link überprüft am 09.04.2018).

24 Vgl. Deutsches Filminstitut, Frankfurt, Zulassungskarte mit Prüf-Nr. 47317 und https:// web.archive.org/web/20180409142820/https://www.filmportal.de/film/altstoff-roh stoff_859d65f639b5428a93eb1103c812a407 (Link überprüft am 09.04.2018).

25 Seit Ende des 19. Jahrhunderts wurden für Feinseifen pflanzliche, meist importabhängige Fette (Palmöl, Kokosöl etc.) verwendet (Deite 1887: 91-93).

26 Im Ersten Weltkrieg waren die Gasmaskenfilter mit Adsorptionskohle gefüllt (Ullmann \& Reitstötter 1930: $617 \mathrm{f}$.).

27 ESM, Abb. 11: Umschlag und Doppelseite eines Taschenkalenders, den der Reichskommissar für Altmaterialverwertung als Belohnung für besonders gute Sammelleistungen verschenkte, 1944, Schulmuseum Bergisch Gladbach. Der Text war allerdings insofern irreführend, als er die Information unterschlug, dass sich Sammelknochen weder zur Herstellung von Fotogelatine noch zur Gewinnung hochwertiger Öle und Fette eigneten.

28 Vgl. die Zeitschriften Das Rohproduktengewerbe (1941), 270; Altmaterial-Wirtschaft (1944), 2.

29 Verordnung über Knochenfett vom 08.07.1936, Reichsgesetzblatt 1936/I, 565.

30 Ausgekochte oder gebratene Knochen enthielten immer noch 8-10 Prozent Fett.

31 Viel deutlicher als die Unterrichtsmaterialien des „Dritten Reichs“ zeigte der BufaFilm (1918), dass die Knochensammlungen militärisch wichtig waren: zur Knochenleimgewinnung für den Flugzeugbau, zur Knochenfettgewinnung für die Seifen-, Torpedoschmieröl-und die Margarinefabrikation sowie zur Glyceringewinnung. Vgl. auch Anm. 17.

32 Vermutlich verbirgt sich Egon Witthuhn auch hinter dem Kürzel E. W. rechts unten auf der Knochenlehrtafel für die Sonderschule, die auffallenderweise ohne dazu gehörenden Artikel in der Zeitschrift Die deutsche Sonderschule publiziert wurde und völlig isoliert in diesem Zeitschriftenband steht (Anonym 1938).

33 ESM, Abb. 12: Lehrtafel aus der DDR zur Propagierung der dort nach Kriegsende weitergeführten Knochensammlungen, ca. 1951. Farblithografie auf Papier, $99 \times 68 \mathrm{~cm}$, Deutsches Historisches Museum, Berlin, P 94/606.

34 ESM, Abb. 13: Von der Propagandaabteilung des Reichskommissars für Altmaterialverwertung kreierter „Knochenstammbaum“ (Kühn 1939: 15). Die drei unteren, tragenden 
Äste des Baumes sind mit „Knochenfett“, „entfettete Knochen“ und „rohes Klauenöl“ beschriftet und suggerieren, dass der wichtigste Inhaltsstoff der Knochen Fette seien. Dies entsprach jedoch ganz und gar nicht dem tatsächlichen prozentualen Anteil der Knocheninhaltsstoffe, in denen lediglich 5-15 Prozent Fett enthalten sind; allerdings zeigt diese Darstellung sehr deutlich, wie dringend das Deutsche Reich angesichts des großen Fettmangels auf die Gewinnung dieses geringen Anteils an Knochenfett angewiesen war. Hätte die Darstellung den tatsächlichen Gegebenheiten entsprochen, so hätten die Futterschrot-, Knochenmehl-, Knochenasche- und Knochenkohleherstellung die tragenden Äste sein müssen. Diese Produkte basierten auf den anorganischen Bestandteilen der Knochen, die mit 50-60 Prozent seine Hauptbestandteile sind; in der vorliegenden Schemazeichnung wurden die Hauptbestandteile dagegen völlig unzutreffend zu unbedeutenden Nebenästen reduziert.

\section{Literatur}

Alimenti, Cesare 1940. La lotta contro gli sprechi ed il ricupero dei rifiuti urbani. Milano: Istituto per gli studi corporativi ed autarchici.

Anonym 1937. Lehrmittel zur unterrichtlichen Behandlung des Vierjahresplans. Schulwart. Berichte über neue Lehrmittel (34) Nr. 2: 29-33.

Anonym 1937a. Wir alle helfen mit! Hilf mit! Illustrierte deutsche Schülerzeitung. Nr. 1 (Oktober): 28 .

Anonym 1938. Zur Knochensammlung in der Sonderschule. Die deutsche Sonderschule (5) Nr. 1: 50.

Anonym 1938a. Eine alte Aufgabe der chemischen Industrie: Verwertung des Wertlosen. Die Chemische Industrie (Gemeinschaftsausgabe) (61) Nr. 5: 97-100.

Anonym 1940. Wir sammeln Knochen. Wir helfen am Aufbau Deutschlands! Schulwart. Lehrmittelführer für das gesamte Schulwesen (37) Nr. 1: 24.

Anonym 1940a. Knochensammlung im Protektorat Böhmen und Mähren. Die chemische Industrie (Nachrichten-Ausgabe) (63) Nr. 2: 27.

Anonym 1940b. Helft den Krieg gewinnen! Hilf mit! Illustrierte deutsche Schülerzeitung (Nr. 7): 111.

Anonym 1942. Schulpreise für Altmaterialsammlung. Der Deutsche Erzieher. Reichszeitung des Nationalsozialistischen Lehrerbundes (Nr. 10): 286.

Anonym 1942a. Allgemeine Anordnung über die Knochensammlung, den Knochenhandel und die Knochenverarbeitung vom 8.8.1942. Gelatine - Leim - Klebstoffe (10) Nr. 9/10: 103-104.

Anonym 1942b. Erfolgreiche Altstoffsammlung. Die deutsche Sonderschule (9) Nr. 2: 78.

Anonym 1942c. Altstoffsammlung der zweiklassigen Hilfsschule in Schwerte-Ruhr im Jahre 1941. Die deutsche Sonderschule (9) Nr. 4: 154-155.

Anonym 1942d. Knochenbewirtschaftung im Generalbezirk Lettland. Die chemische Industrie (Nachrichten-Ausgabe) (65) Nr. 43/44: 456.

Anonym 1942e. Vorbildliche Altstoffsammlung. Die deutsche Sonderschule (9) Nr. 5/6: 203.

Anonym 1942f. Erfassung von Knochen im Ostland. Die chemische Industrie (NachrichtenAusgabe) (65) Nr. 27/28: 285.

Anonym 1942g. Bewirtschaftung von Knochen und Hörnern. Die chemische Industrie (Nachrichten-Ausgabe) (65) Nr. 27/28: 285.

Anonym 1942h. Schulvorsammelstellen - Auszeichnung der besten Schüler und Schülerinnen. Amtliches Schulblatt für den Regierungsbezirk Kattowitz (3) Nr. 17 (11.6.1942): $113-114$.

Anonym 1942i. Slowakei. Die chemische Industrie (Nachrichten-Ausgabe) (65) Nr. 27/28: 287.

Anonym 1942j. Mutti, Bärbel und das Altmaterial. Der Deutsche Erzieher. Reichszeitung des Nationalsozialistischen Lehrerbundes (Nr. 1): 17. 
Anonym 1943. Eine Million Bleistifte für die Schulaltstoffsammler. Marburger Zeitung (25.1.1943): 6

Anonym 1943a. Altstofferfassung in den Schulen. Amtliches Schulblatt für den Regierungsbezirk Kattowitz (4) Nr. 34 (1.12.1943): 196.

Anonym 1943b. Sammlung von Knochen in Kroatien. Gelatine - Leim - Klebstoffe (11) Nr. 3/4: 36.

Anonym 1943c. Altstoffsammlung. Amtliches Schulblatt für den Regierungsbezirk Kattowitz (4) Nr. 18 (21.6.1943): 123.

Anonym 1943d. Erfassung von Altstoffen durch die Schulen. Amtliches Schulblatt für den Regierungsbezirk Kattowitz (4) Nr. 26 (11.9.1943): 160-161.

Anonym 1944. Die Lage auf dem Altstoffmarkt. Wohnen (19) Nr. 7: 123.

Anonym 1946. Knochen - eine landeseigene Rohstoffquelle. Wohnen (21) Nr. 2: 29.

Baier, Wolfgang 1977. Quellendarstellungen zur Geschichte der Fotografie. München: Schirmer/Mosel.

Barwich, Brunhild 2003. Rosinen und Sauerkraut, Erinnerungen an Kindheit und Jugend 1930-1948. Wittenberg: Drei Kastanien.

Bäumer-Schleinkofer, Änne 1994. Wissenschaftsgeschichte als Mittel der politischen Indoktrination: Physik- und Chemiegeschichte im Schulunterricht im Dritten Reich. In: Christoph Meinel und Peter Voswinckel (Hg.). Medizin, Naturwissenschaft, Technik und Nationalsozialismus. Kontinuitäten und Diskontinuitäten. Stuttgart: Verlag für Geschichte der Naturwissenschaften und der Technik: 282-294.

Bäumer-Schleinkofer, Änne 1995. Nazi Biology and Schools. Frankfurt/M.: Peter Lang.

Beier, Ilse 1934. Zur Schulreform. Chemieunterricht und Erziehung im Dritten Reich. Unterrichtsblätter für Mathematik und Naturwissenschaften (40): 252-254.

Berg, Anne 2015. The Nazi Rag-Pickers and their Wine: The Politics of Waste and Recycling in Nazi Germany. Social History (4): 446-472.

Bergmann, Eugen 1919. Gelatine und Leim. In: Fritz Ullmann (Hg.). Enzyklopädie der technischen Chemie. Bd. 6: Gasfilter - Kautschuke, künstliche. Berlin/Wien: Urban \& Schwarzenberg: 28-53.

Brämer, Rainer und Armin Kremer 1980. Physikunterricht im „Dritten Reich“. Marburg/L.: Soznat.

Braun, Adee 2014. Turning Bacon into Bombs: The American Fat Salvage Committee. The Atlantic. 18.4.2014. URL: https://web.archive.org/web/20180409141948/https://www. theatlantic.com/health/archive/2014/04/reluctantly-turning-bacon-into-bombs-dur ing-world-war-ii/360298/ (09.04.2018).

Brockmann, Anne 1994. Altmaterialsammlung. In: Margret Birckenbach, Anneliese Kaut, Meta Techam, Christa Erich, Lisa Schomburg und Anne Brockmann (Hg.). Frauen blicken zurück. Erinnerungen an die Kriegsjahre 1939-1945, Hamburg: Klönschnack: 121-122.

Büdingen, Maximilian Graf von 1938. Aus alt wird neu. Schuljugend hilft am Vierjahresplan. Hilf mit! Illustrierte deutsche Schülerzeitung (Nr. 4): 123.

Bullus, Jacob 2017. Salvage on the Home Front. Royal Voluntary Service Archive E Heritage Collection. URL: https://web.archive.org/web/20180409142242/https://www. royalvoluntaryservice.org.uk/Uploads/Documents/About\%20us/Salvage_on_the_ Home_Front_May2017.pdf (09.04.2018).

Deite, Carl 1887. Handbuch der Seifenfabrikation. Berlin: Julius Springer.

Denton, Chad 2013. ,Récupérez!' The German Origins of French Wartime Salvage Drives, 1939-1945. Contemporary European History (22) Nr. 3: 399-430.

Denton, Chad 2014. Steel of Victory, Scrap of Defeat: Mobilizing the French Home Front, 1939-40. War E Society (33) Nr. 2: 98-130.

Denton, Chad 2016. Entre résidus et ressources. René Martin et le traitement des déchets à Lyon (1939-1945). In: Isabelle von Bueltingsloewen, Laurent Douzou, Jean-Dominique Durand, Hervé Joly und Jean Solchany (Hg.). Lyon dans la Seconde Guerre mondiale. Villes et métropoles à l'épreuve du conflit. Rennes: Presses universitaires: 135-146.

Dörmer, Karl-Edmund und Ludwig Dörmer (Bearb.) 1939. Arendt-Dörmer. Grundzüge der Chemie für den Unterricht an höheren Schulen. Bd. 2 A, Ausgabe für Jungen (Klasse 6-8). Leipzig: Johann Ambrosius Barth. 
Dorner, Adolf 1935. Mathematik im Dienste der nationalpolitischen Erziehung mit Anwendungsbeispielen aus Volkswissenschaft, Geländekunde und Naturwissenschaft. 2. Aufl. Frankfurt/M.: Moritz Diesterweg.

Durflinger, Serge Marc 2006. Fighting from home. The Second World War in Verdun/Quebec. Vancouver/Toronto: UBC Press.

Erziehung und Unterricht in der Höheren Schule 1938. Amtliche Ausgabe des Reichs- und Preußischen Ministeriums für Wissenschaft, Erziehung und Volksbildung. Berlin: Weidmann.

Facharbeitsgemeinschaft deutscher Erzieher (Hg.) 1940. Fibel für die deutsche Jugend. Berlin: Deutscher Schulbuchverlag.

Fleck, Hugo 1878. Die Fabrikation chemischer Producte aus thierischen Abfällen. 2. Aufl. Braunschweig: Friedrich Vieweg und Sohn.

Garz, Paul 1939. Wir sammeln Knochen. Frankfurt/M.: Moritz Diesterweg.

Gerhardt, Hans und Albert Höfner 1942. Deutsche Roh-und Werkstoffe mit Bilderatlas durch die deutsche Rohstoffwirtschaft. 3. Aufl. Frankfurt/M.: Fritz Knapp.

Gerngross, Otto und E. Goebel 1933. Chemie und Technologie der Leim- und Gelatine-Fabrikation. Dresden/Leipzig: Theodor Steinkopff.

Gölz, Klaus und Walter Jansen 1990. Der Chemieunterricht im NS-Staat - Ein Beitrag zur Geschichte der Chemiedidaktik. Mitteilungen Gesellschaft Deutscher Chemiker, Fachgruppe Geschichte der Chemie (4): 23-36.

Gölz, Klaus und Walter Jansen 1993. Der Chemieunterricht im NS-Staat - Ein Beitrag zur Geschichte der Chemiedidaktik. Naturwissenschaften im Unterricht - Chemie (4) Nr. 17: 48-51.

Grüntzig, Willmar 1939. Schulversuche zur Küchenchemie. Leipzig: Dürr.

Haferkorn, Walter, 1938. Schulversuche mit deutschen Roh-und Werkstoffen. Leipzig: „Kultur" Verlag für Lehrmittel.

Haferkorn, Walter und Herbert Priemer 1937-1939. Anschauungstafeln zur deutschen Nationalwirtschaft. Leipzig: „Kultur“ Verlag für Lehrmittel.

Hartmann, Joseph 1938. Der Vierjahresplan im Schulunterricht. Eine Stoffdarbietung für den Unterricht. 3. Aufl. Osterwiek/Berlin: A. W. Zickfeldt.

Hertenstein, Paul 1939. Altmaterialwirtschaft im Vierjahresplan. Der Deutsche Erzieher. Reichszeitung des Nationalsozialistischen Lehrerbundes (Nr. 12): 278-281.

Hessenland, Max 1938. Praktikum der gewerblichen Chemie. München/Berlin: J. F. Lehmann.

Irving, Henry 2016. Paper Salvage in Britain during the Second World War. Historical Research (89) Nr. 244: 373-393.

Kahn, Fritz 1923/24. Industrieprodukte des Knochens. Jahrbuch der Technik (10): 14-18.

Keller, Fritz 2015. Die Küche im Krieg. Lebensmittelstandards 1933 bis 1945. Wien: new academic press.

Kimble, James J. 2014. Prairie Forge. The Extraordinary Story of the Nebraska Scrap Metal Drive of World War II. Lincoln/London: University of Nebraska Press.

Kinttof, Walter 1935. Schulversuche zur Chemie der Kampfstoffe. Ein Experimentierbuch zum Gas- und Luftschutz. Berlin: Carl Heymanns.

Köstering, Susanne 1997. „Pioniere der Rohstoffbeschaffung“. Lumpensammler im Nationalsozialismus, 1934-1939. WerkstattGeschichte (17): 45-65.

Köstering, Susanne 2003. „Millionen im Müll?“ Altmaterialverwertung nach dem Vierjahresplan. In: Susanne Köstering und Renate Rüb (Hg.). Müll von Gestern? Eine umweltgeschichtliche Erkundung in Berlin und Brandenburg. Münster u. a.: Waxmann: 139-149.

Krause, R. 1943. Schulaltstoffsammlung - weder schulfremd noch schulstörend! Der Deutsche Erzieher. Reichszeitung des Nationalsozialistischen Lehrerbundes (Nr. 7/8): 143-145.

Kremer, Gabriele 2011. Die Sonderschule im Nationalsozialismus: das Beispiel Hilfsschule. In: Klaus-Peter Horn und Jörg-W. Link (Hg.). Erziehungsverhältnisse im Nationalsozialismus. Totaler Anspruch und Erziehungswirklichkeit. Bad Heilbrunn: Julius Klinkhardt: 163-184.

Kühn, Helmut 1939. Jeder muß helfen! Deine Pflichten im Vierjahresplan. Eine lehrreiche Unterhaltung. Berlin: Köhler. 
Kühn, Michael 1998. Unterrichtsfilm im Nationalsozialismus. Die Arbeit der Reichsstelle für den Unterrichtsfilm/Reichsanstalt für Film und Bild in Wissenschaft und Unterricht. Mammendorf/Obb.: septem artes.

Landesbildstelle Württemberg (Hg.) 1943. Filme und Lichtbilder für den Unterricht der allgemeinbildenden Schulen sowie der landwirtschaftlichen Berufsschulen für Mädchen (Hauswirtschaftl. Berufsschulen). Stuttgart: Gebrüder Knöller.

Liebig, Justus von 1861. Rede in der öffentlichen Sitzung der kgl. Akademie der Wissenschaften am 28.11.1861 zur Feier des Allerhöchsten Geburtsfestes Sr. Majestät des Königs Maximilian II. München: Kgl. bayr. Akademie.

Lübke, Anton 1938. Das deutsche Rohstoffwunder. Wandlungen der deutschen Rohstoffwirtschaft. Stuttgart: Verlag für Wirtschaft und Verkehr, Forkel \& Co.

Metzner, Karl 1931. Staatsbürgerkunde durch Mathematik und Naturwissenschaften. Grundsätzliches, Wege und Ziele. Leipzig: Quelle \& Meyer.

Meyer, Erich 1939. Wir sammeln Knochen! Text zum „Schulmann“-Bild Nr. 260: Die Verwertung des Knochens. Bearbeitet im Einvernehmen mit dem Reichsausschuss für volkswirtschaftliche Aufklärung. Der praktische Schulmann (Nr. 7/8): 98-100.

Miller, Gordon von 2014. Bedeutung und Erscheinungsformen wirtschaftlicher Betätigung von Schülern. Studien zum Schul- und Bildungsrecht (9): 29-63.

Morgner, Walther 1935. Schulversuche zum Luftschutz. Leipzig: Dürr.

Mosby, Ian 2014. Food will win the war: the politics, culture, and science offood on Canada's home front. Vancouver/Toronto: UBC Press.

Müller, Rolf-Dieter 1991: Die deutsche Wirtschaftspolitik in den besetzten Gebieten 19411943. Der Abschlußbericht des Wirtschaftsstabes Ost und Aufzeichnungen eines Angehörigen des Wirtschaftskommandos Kiew. Boppard/Rhein: Boldt. URL: http://ostdok.

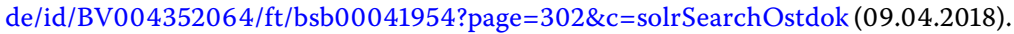

Müller, Walter 1997: Schulwandbilder als Quellen schul- und bildungshistorischer Forschung. In: Hanno Schmitt, Jörg-W. Link und Frank Tosch (Hg.). Bilder als Quellen der Erziehungsgeschichte. Bad Heilbrunn/Obb.: Julius Klinkhardt: 189-217.

Nadolni, Barbara 1944. Unerschöpfliche Rohstoffquelle. Der oberschlesische Wanderer, Amt. Blatt der NSDAP und aller Behörden (117) Nr. 141 (23.5.1944).

Nagel, Anne C. 2012. Hitlers Bildungsreformer. Das Reichsministerium für Wissenschaft, Erziehung und Volksbildung 1934-1945. Frankfurt/M.: S. Fischer.

Neubert, Johannes 1940. Der Einsatz der Hilfsschule bei der Sammlung von Altmaterial. Die deutsche Sonderschule (7) Nr. 5/6: 227-228.

Neubert, Johannes 1941. Hilfsschüler sammeln Altstoffe. Die deutsche Sonderschule (8) Nr. 5: 264-268.

Niethammer, Verena 2016. Indoktrination oder Innovation? Der Unterrichtsfilm als neues Lehrmedium im Nationalsozialismus. Journal of Educational Media, Memory, and Society (8) Nr. 1: 30-60.

Oldenziel, Ruth und Milena Veenis 2001. De oorlogseconomie: zuinigheid en noodgedwongen vindingrijkheid,1940-1945. In: Techniek in Nederland in de twintigste eeuw. Bd. 4: Huishouden, medische techniek. Zutphen: Walburg Pers: 83-101.

Ost, Hermann 1942. Lehrbuch der Chemischen Technologie. 23. Aufl. Leipzig: Max Jänecke.

Ostertag, Robert von, Erich Moegle und Siegfried Braun 1958. Die Tierkörperbeseitigung. 2. Aufl. Berlin/Hamburg: Paul Parey.

Pelzer, Birgit und Reinhold Reith 2001. Margarine. Die Karriere der Kunstbutter. Berlin: Klaus Wagenbach.

Perrun, Jody 2009. The Spirit of Service: Winnipeg's Voluntary War Services During the Second World War. In: Esyllt W. Jones und Gerald Friesen (Hg.). Prairie Metropolis. New Essays on Winnipeg Social History. Winnipeg: University of Manitoba Press: 160186.

Petzold, Rudolf 1941. Die Altstoffe und die Rohstoffversorgung Deutschlands. Borna/Leipzig: Robert Noske.

Pricks, Hellmuth 1937. Einfache Lehrversuche mit Aluminium und seinen Legierungen. Berlin: Aluminium-Zentrale.

Pröbsting, G. 1936. Zur Behandlung der Chemie des Photographierens in der Schule. Film und Bild in Wissenschaft, Erziehung und Unterricht (2) Nr. 7: 230-231. 
Reichsanstalt für Film und Bild in Wissenschaft und Unterricht (Hg.) 1940. Die Unterrichtsfilme der Allgemeinbildenden Schulen. Volksschule - Mittelschule - Höhere Schule. Stuttgart/Berlin: W. Kohlhammer.

Reichskommissar für Altmaterialverwertung (Hg.) 1938a. „Der Knochen als Rohstoff und seine Verwertung". Erläuterungsheft zur Anschauungskarte, bearbeitet von H. E. Witthuhn. Berlin: Dr. v. Arnim \& Co.

Reichskommissar für Altmaterialverwertung (Hg.) 1938b. Der Knochen als Rohstoff und seine Verwertung. Lichtbild-Reihe L 12, Bildband Nr. 419. Berlin: Deutsche Lichtbild-Gesellschaft.

Reichsminister für Wissenschaft, Erziehung und Volksbildung 1938. Knochenlehrkarte. Erlaß vom 5.9.1938. Deutsche Wissenschaft, Erziehung und Volksbildung (4): 430-431.

Reichsminister für Wissenschaft, Erziehung und Volksbildung 1939. Sammlung von Knochen und Altmaterial in den Schulen. Erlaß vom 24.11.1939. Deutsche Wissenschaft, Erziehung und Volksbildung (5): 594.

Reichsminister für Wissenschaft, Erziehung und Volksbildung 1941. Altstofferfassung durch die Schuljugend. Runderlaß vom 21.6.1941. Deutsche Wissenschaft, Erziehung und Volksbildung (7): 261-262.

Reichsminister für Wissenschaft, Erziehung und Volksbildung 1945. Einsatz der Schulen zur Altmaterialerfassung. Erlaß vom 22.1.1945. Deutsche Wissenschaft, Erziehung und Volksbildung (11), Nr. 3-5: 14.

Reichswaltung des NS-Lehrerbundes (Hg.) o.D. [um 1940]. Rohstoff Knochen. LehrschauBogen Nr. 18 und 18a. Berlin-Tempelhof: H. A. Braun \& Co. Institut für Zeitgeschichte, München, Archiv Z_1482_0018.

Reith, Birgit 2002. „Fett aus Kohle“? Die Speisefettsynthese in Deutschland 1933-1945. Technikgeschichte (69) Nr. 3: 173-205.

Reith, Reinhold 2010. Kohle, Strom und Propaganda im Nationalsozialismus: Die Aktion „Kohlenklau“. In: Theo Horstmann und Regina Weber (Hg.). „Hier wirkt Elektrizität“. Werbung für Strom 1890 bis 2010. Essen: Klartext: 142-159.

Rothenbach-Abderhalden, Adèle 1948. Die Arbeit der Altstoff-Frauen des zivilen Frauenhilfsdienstes der Stadt Olten. Oltner Neujahrsblätter (6): 19-26.

Sagemüller, Fritz 1994. Erler Schulchronik 1933-44. Raesfeld-Erle: Heimatverein Erle.

Scharf, Reinhold und Hans Golombeck 1940. Unsere Kleidung. Schulversuche über Faserstoffe, Spinnerei und Weberei, Farbstoffe und Färberei. Frankfurt/M.: Otto Salle.

Schiffner, Adolf 1944. Rechenbuch für Volksschulen. Gaue Westfalen-Nord u. Süd. Ausgabe $B$ für wenig gegliederte Schulen. Heft 5 - Siebentes und achtes Schuljahr. Leipzig: W. Crüwell.

Schildt, Axel 2005. Jenseits der Politik? Aspekte des Alltags. In: Forschungsstelle für Zeitgeschichte in Hamburg (Hg.). Hamburg im „Dritten Reich“. Göttingen: Wallstein: 249-304.

Schlenker, Ernst 1932. Das Glycerin. Stuttgart: Wissenschaftliche Verlagsgesellschaft.

Schulze, Friedrich 1938. Lehrversuche mit Fasern und Geweben. Berlin: Carl Heymanns.

Schwierskott, Konrad 1938. Die deutsche Schule im Zeichen des Vierjahresplanes. Weltanschauung und Schule (2): 550-555.

Das Spezial-Archiv der deutschen Wirtschaft (Hg.) 1931. Die grossen Chemie-Konzerne Deutschlands 1931. Berlin: Verlag R. \& H. Hoppenstedt.

Staufenbiel, Georg 1936. Lehrversuche mit den Baustoffen Stein, Mörtel, Beton. Berlin: Carl Heymanns.

Steinberg, Günther Karl 2008. Eine Geschichte der Heimkehr. Mein Leben Teil I, 1929-1956. Norderstedt: Books on Demand.

Stohmann, Friedrich und Carl Engler 1872. Handbuch der technischen Chemie. Nach A. Payen's Chimie industrielle frei bearbeitet. Bd. 1: Anorganische Chemie. Stuttgart: E. Schweizerbart.

Stohmann, Friedrich und Carl Engler 1874. Handbuch der technischen Chemie. Nach A. Payen's Chimie industrielle frei bearbeitet. Bd. 2: Organische Chemie. Stuttgart: E. Schweizerbart.

Stoya, Eva-Maria 2002. Chemieunterricht in der NS-Zeit. Frankfurt u. a.: Peter Lang.

Strasser, Susan 2013. Complications and Complexities: Reflections on Twentieth-Century European Recycling. Contemporary European History (22): 517-526. 
Teweleit, Manfred 1993. Ostwind. Leben in Ostpreußen. Flucht aus Memel über Westpreußen nach Pommern. Die Tragödie 1944-1947. Gütersloh: Bonewie.

Thomsen, Winfried 1943. Betrachtungen zur Altstoffsammlung. Der Deutsche Erzieher. Reichszeitung des Nationalsozialistischen Lehrerbundes (Nr. 2): 39-40.

Thorsheim, Peter 2013: Salvage and Destruction: The Recycling of Books and Manuscripts in Great Britain during the Second World War. Contemporary European History (22): 431-452.

Thorsheim, Peter 2015. Waste into Weapons. Recycling in Britain during the Second World War. Cambridge: Cambridge University Press.

Ullmann, Fritz und Josef Reitstötter 1930. Entfärbungs- und Adsorptionskohlen. In: Fritz Ullmann (Hg.). Enzyklopädie der technischen Chemie. 2. Aufl. Bd. 6: Gold - Kühler. Berlin/Wien: Urban \& Schwarzenberg: 617-624.

Ungewitter, Claus (Hg.) 1938. Verwertung des Wertlosen. Berlin: Wilhelm Limpert.

Vogel, August 1874. Justus Freiherr von Liebig als Begründer der Agrikultur-Chemie. Eine Denkschrift. München: Kgl. bayr. Akademie.

Vorländer, Herwart 2000. Weißer Jahrgang. Meine Zeit, damals‘ - und später. Düsseldorf: Archiv der Evangelischen Kirche im Rheinland.

Wagner, Johannes Rudolf 1868. Die chemische Technologie als Leitfaden bei Vorlesungen. 7. Aufl. Leipzig: Otto Wigand.

Weber, Heike 1999. Technikkonzeptionen in der populären Sachbuchliteratur des Nationalsozialismus. Die Werke von Anton Zischka. Technikgeschichte (66) Nr. 3: 205-236.

Weber, Heike 2013a. La politique des déchets ménagers en Allemagne au XXe siècle. Pour mémoire. Revue du Ministère de l'écologie, du développement durable et de l'énergie (12): 139-151.

Weber, Heike 2013b. Towards “Total” Recycling: Women, Waste and Food. Waste Recovery in Germany, 1914-1939. Contemporary European History (22): 371-397.

Weber, Heike 2014. Den Stoffkreislauf am Laufen halten. Restearbeit und Resteökonomie des 20. Jahrhunderts. In: Kijan Espahangizi und Barbara Orland (Hg.). Stoffe in Bewegung. Beiträge zu einer Wissensgeschichte der materiellen Welt. Zürich/Berlin: diaphanes: $145-171$.

Weber, Heike 2019 (in Vorbereitung). Reste und Recycling bis zur "grünen Wende“ - Eine Stoff- und Wissensgeschichte alltäglicher Abfälle. Göttingen: Vandenhoek \& Ruprecht.

Weber, Heike und Chad Denton 2018 (im Erscheinen). Collaborating in the Nazi Recycling Project: Bone Collections in Germany and France during the Second World War. In: Tatjana Tönsmeyer (Hg.). Coping with Hunger and Shortage under German Occupation in World War II. Basingstoke: Macmillan.

Weber, Wilhelm 1937. Der chemisch-technologische Film. Film und Bild in Wissenschaft, Erziehung und Volksbildung (3): 273-277.

Weiss, Sheila Faith 1994. Pedagogy, Professionalism and Politics: Biology Instruction during the Third Reich. In: Monika Renneberg und Mark Walker (Hg.). Science, Technology and National Socialism. Cambridge: Cambridge University Press: 184-196.

Welsch, Fritz 1981. Geschichte der chemischen Industrie. Abriß der Entwicklung ausgewählter Zweige der chemischen Industrie von 1800 bis zur Gegenwart. Berlin (Ost): VEB Deutscher Verlag der Wissenschaften.

Wimmer 1937. Der Vierjahresplan in der Schule. Volksaufklärung und Schule. Informationsdienst für die gesamte Lehrerschaft (28): 4-8.

Wolter, Friedrich 1938. Der Vierjahresplan in der Schularbeit. 2. Aufl. Leipzig: Julius Klinkhardt.

Wüstenfeld, Gustav Adolf 2002. Lumpen, Eisen, Knochen und Papier, ja das sammeln wir .... industrie-kultur (3): 29.

Wüstner, Hermann 1938. Chemisch-technologische Versuche im Rahmen des Vierjahresplanes. Praktische Schulchemie (3) Nr. 1: 1-4.

Zeitler, Hans 1940. Knochen als Rohstoff, ein Thema für eine chemische Arbeitsgemeinschaft. Unterrichtsblätter für Mathematik und Naturwissenschaften (46): 7-11 und $17-23$.

Ziegelmayer, Wilhelm 1936. Rohstoff-Fragen der deutschen Volksernährung. Eine Darstellung der ernährungswirtschaftlichen und ernährungswissenschaftlichen Aufgaben unserer Zeit. Dresden/Leipzig: Theodor Steinkopff. 
Ziegelmayer, Wilhelm 1941. Rohstoff-Fragen der deutschen Volksernährung. Eine Darstellung der ernährungswirtschaftlichen und ernährungswissenschaftlichen Aufgaben unserer Zeit mit einem Ausblick auf die Großraumwirtschaft. 4. Aufl. Dresden/Leipzig: Theodor Steinkopff.

Zimring, Carl A. 2009. Cash for your trash: scrap recycling in America. New Brunswick: Rutgers University Press.

Elisabeth Vaupel

Forschungsinstitut

Deutsches Museum

80306 München

Deutschland

e.vaupel@deutsches-museum.de

Florian Preiß

Bibliothek

Deutsches Museum

80306 München

Deutschland

f.preiss@deutsches-museum.de 